\title{
On Hilbert's 17th problem and Pfister's multiplicative formulae for the ring of real analytic functions
}

\author{
Francesca ACQUistapace, FAbrizio Broglia And José F. FERnAndo
}

\begin{abstract}
In this work, we present "infinite" multiplicative formulae for countable collections of sums of squares (of meromorphic functions on $\mathbb{R}^{n}$ ). Our formulae generalize the classical Pfister's ones concerning the representation as a sum of $2^{r}$ squares of the product of two elements of a field $K$ which are sums of $2^{r}$ squares. As a main application, we reduce the representation of a positive semidefinite analytic function on $\mathbb{R}^{n}$ as a sum of squares to the representation as sums of squares of its special factors. Recall that roughly speaking a special factor is an analytic function on $\mathbb{R}^{n}$ which has just one complex irreducible factor and whose zeroset has dimension between 1 and $n-2$.
\end{abstract}

Mathematics Subject Classification (2010): 11E25 (primary); 26E05, 32C07, 12D15, 14P20 (secondary).

\section{Introduction}

It is well known that the ring of analytic functions on a real analytic manifold $M$ is far from being a unique factorization domain because factorization involves, in the non compact case, infinitely many factors and if $H^{1}\left(M, \mathbb{Z}_{2}\right) \neq 0$ there are analytic functions on $M$ which admit different factorizations (see [21]). However, if we focus on $\mathbb{R}^{n}$, we are able to associate to a real analytic function on $\mathbb{R}^{n}$ a (possibly infinite) collection of irreducible factors (see Section 6.2), unique up to analytic units, and we provide some procedure involving sheaf theory (via a sheaf-product, see Section 2.3) to recover the initial analytic function from its irreducible factors. Thus, we manage an additional tool: factorization, and one of the main purposes of this work is to reduce the representation of an analytic function $f: \mathbb{R}^{n} \rightarrow \mathbb{R}$ as a sum of squares (of meromorphic functions on $\mathbb{R}^{n}$ ) to the representation of the irreducible factors of $f$ which divide it with odd multiplicity. This involves the development of “infinite" Pfister's multiplicative formulae for the field of meromor-

First and second authors supported by Italian GNSAGA of INdAM and MIUR. All authors supported by Spanish GAAR MTM2011-22435. Third author also supported by GAAR Grupos UCM 910444.

Received January 9, 2012; accepted in revised form March 29, 2012. 
phic functions on $\mathbb{R}^{n}$. Such approach generalizes the classical result concerning the representation of analytic function with discrete zeroset as sum of squares provided in [5], which was our starting point for the study of Hilbert's 17th problem in the analytic case (see $[2,8]$ ). Of course, the zero sets of the irreducible factors of an analytic function with discrete zeroset are singletons, which is obviously the simplest possible case.

Since we are concerned with the analytic setting, the involved sum of squares might be infinite. More precisely, the representation of a positive semidefinite analytic function on $\mathbb{R}^{n}$ as an infinite sum of squares (of meromorphic functions on $\mathbb{R}^{n}$ ) consists in finding a non identically zero analytic function $g$ such that $g^{2} f$ is an infinite sum of squares $\sum_{i \geq 1} a_{i}^{2}$ of analytic functions which converges in the following strong sense: there is an open neighborhood $\Omega$ of $\mathbb{R}^{n}$ in $\mathbb{C}^{n}$ to which all involved data $f, g, a_{i}$ extend to holomorphic functions $F, G, A_{i}$ and the series $\sum_{i \geq 1} A_{i}^{2}$ converges to $G^{2} F$ in the ring $\mathcal{H}(\Omega)$ of holomorphic functions on $\Omega$ endowed with the usual compact-open topology (see Definition 2.10 for further details). Observe that in an infinite representation as sum of squares the "denominator" is the same for all the summands of the series. Clearly, this situation can be always achieved for finite sum of squares finding a common denominator of such representation. In what follows, $\left\{*_{k}\right\}_{k \geq 1}$ denotes a collection of objects $*_{k}$ which is either countable or finite but we are not interested in the precise number of its elements. In this framework our main result is the following:

Theorem 1.1 (Analytic Pfister's formulae). Let $\left\{f_{k}\right\}_{k \geq 1}$ be a collection of positive semidefinite analytic functions on $\mathbb{R}^{n}$ such that the family $\left\{X_{k}=\left\{f_{k}=0\right\}\right\}_{k \geq 1}$ of their zero sets is locally finite. Let $f$ be a positive semidefinite sheaf-product of the functions $f_{k}$, that is, a positive semidefinite analytic function on $\mathbb{R}^{n}$ such that

$$
f \mathcal{O}_{\mathbb{R}^{n}, x}=\left\{\begin{array}{cl}
\prod_{k \geq 1, x \in X_{k}} f_{k, x} \mathcal{O}_{\mathbb{R}^{n}, x} & \text { if } x \in \bigcup_{k \geq 1} X_{k}, \\
\mathcal{O}_{\mathbb{R}^{n}, x} & \text { otherwise. }
\end{array}\right.
$$

Then,

(i) If each $f_{k}$ is a finite sum of squares, $f$ is an infinite sum of squares.

(ii) If each $f_{k}$ is a sum of $2^{r}$ squares (for a fixed $r$ ), $f$ is a sum of $2^{n+r}$ squares.

(iii) If each $f_{k}$ is an infinite sum of squares, there is a positive semidefinite analytic function $q$ on $\mathbb{R}^{n}$ such that $\{q=0\} \subset\{f=0\}, \operatorname{dim}\{q=0\}<\operatorname{dim}\{f=0\}$ and $q f$ is an infinite sum of squares.

We say that an analytic function $f: \mathbb{R}^{n} \rightarrow \mathbb{R}$ is of Nash type if there is an open neighborhood $W$ of $\{f=0\}$ in $\mathbb{R}^{n}$ and an analytic diffeomorphism $\varphi$ from an (affine) Nash manifold $M$ to $W$ such that $f \circ \varphi$ is the product of a Nash function on $M$ times an analytic unit. Recall that a Nash manifold $M$ is a semialgebraic subset of some $\mathbb{R}^{m}$ which is in addition an analytic submanifold of $\mathbb{R}^{m}$; a Nash function $f$ on $M$ is an analytic function with semialgebraic graph (see [4, Section 8] for further details). As first consequences of Theorem 1.1, [14,20] and [4, 8.5.6] we have the following two results. 
Theorem 1.2. Let $\left\{f_{k}\right\}_{k \geq 1}$ be a collection of positive semidefinite analytic functions on $\mathbb{R}^{n}$ such that the family $\left\{X_{k}=\left\{f_{k}=0\right\}\right\}_{k \geq 1}$ of their zero sets is locally finite and each $X_{k}$ is either compact or of Nash type. Let $f$ be a positive semidefinite sheaf-product of the functions $f_{k}$. Then, $f$ is an infinite sum of squares.

Theorem 1.3. Let $\left\{f_{k}\right\}_{k \geq 1}$ be a collection of positive semidefinite analytic functions on $\mathbb{R}^{n}$ such that the family $\left\{X_{k}=\left\{f_{k}=0\right\}\right\}_{k \geq 1}$ of their zero sets is locally finite and each $f_{k}$ is of Nash type. Let $f$ be a positive semidefinite sheaf-product of the functions $f_{k}$. Then, $f$ is a sum of $2^{n+1}$ squares.

Theorems 1.2 and 1.3 can be understood as natural but substantial generalizations of the classical results [5, Theorem 1] and [15, Section 4, Theorem 2]; once more, both classical results can be obtained as a new application of Theorem 1.1 (see Section 6). We summarize the statements of such classical results as follows:

Theorem 1.4. Let $f$ be a positive semidefinite analytic function on $\mathbb{R}^{n}$ whose zero set is the union of a discrete set and a compact set. Then, $f$ is a finite sum of squares.

The representation of positive semidefinite functions on a real variety as a sum of squares has attracted the attention of many specialists in number theory, quadratic forms, real algebra and real geometry. This problem goes back to the celebrated Hilbert's 17th problem for polynomial functions. Its solution by E. Artin in 1927 (see [3]) was the starting point for the development of the theory of the real spectrum of a ring, introduced by M. Coste and M.-F. Roy and which has been the crucial technique to approach many problems in Real Geometry (for more details see [4]). The real spectrum has been often used to approach Hilbert's 17th problem in "finitary situations": polynomial functions, Nash functions, analytic function germs at points or at compact sets,... but it has fallen short to deal with analytic functions on $\mathbb{R}^{n}$ for dimension $n \geq 3$ without some compactness assumptions (see $[14,20]$ ). Hilbert's 17th problem for analytic functions asks whether:

H17. Every positive semidefinite analytic function $f: \mathbb{R}^{n} \rightarrow \mathbb{R}$ is a finite sum of squares (of meromorphic functions).

In fact, we show in [2], that a positive solution to H17 above implies the finiteness of the Pythagoras number $p\left(\mathbb{R}^{n}\right)$ of the field $\mathcal{M}\left(\mathbb{R}^{n}\right)$ of meromorphic functions on $\mathbb{R}^{n}$; recall that $p\left(\mathbb{R}^{n}\right)$ is either the least integer $p$ such that each finite sum of squares in $\mathcal{M}\left(\mathbb{R}^{n}\right)$ can be represented as a sum of $p$ squares in $\mathcal{M}\left(\mathbb{R}^{n}\right)$ or $+\infty$ if such an integer does not exist. This reveals that in the analytic setting there is a strong connection between the qualitative and the quantitative aspects of H17, which seems to not appear in the "finitary situations" commented above. Furthemore, if we were able to represent irreducible positive semidefinite analytic functions as sums of squares of meromorphic functions, the real Nullstellensatz proved in [1] in terms of nonnegative functions would get a formulation in terms of the real radical as in the polynomial case. Thus, obstructions to get H17 or to get Risler's Nullstellensatz are essentially the same in the real analytic setting. 
Clearly, infinite sums of squares are positive semidefinite, and this allows us to weaken the classical formulation $\mathrm{H} 17$ and ask whether:

$\mathbf{H 1 7}_{\infty}$. Every positive semidefinite analytic function $f: \mathbb{R}^{n} \rightarrow \mathbb{R}$ is an infinite sum of squares (of meromorphic functions).

As one realizes immediately, Theorem 1.1 reduces $\mathrm{H} 17_{\infty}$, by means of the obvious inductive process and Theorem 1.4 for the initial step, to represent positive semidefinite irreducible analytic functions as infinite sum of squares, and H17 to represent positive semidefinite irreducible analytic functions as finite sum of squares and to prove the finiteness of the Pythagoras number $p\left(\mathbb{R}^{n}\right)$ of the field $\mathcal{M}\left(\mathbb{R}^{n}\right)$.

The article is organized as follows. In Section 2 we collect some constructions and preliminary results that will be used several times in the subsequent sections. Next, Section 3 is devoted to prove Theorems 1.1(ii) and 1.3, while in Section 4 we approach the proof of Theorem 1.1(i) and in Section 5 we demonstrate Theorem 1.1(iii). Finally, Section 6 is dedicated to present some further applications of Theorem 1.1; the main one concerns the reduction of the representation of a positive semidefinite analytic function on $\mathbb{R}^{n}$ as a sum of squares to the representation as sums of squares of its special factors. We also show how analytic Pfister's formulae can be generalized for an arbitrary (affine) analytic manifold and we point out that all the results developed in this article, even those concerning factorization, run, almost in the same way, for any analytic manifold $M$ with $H^{1}\left(M, \mathbb{Z}_{2}\right)=H^{2}(M, \mathbb{Z})=0$.

\section{Preliminaries}

Although we deal with real analytic functions, we will of course use some complex analysis. All through this work holomorphic functions will refer to the complex case and analytic functions to the real case. For further readings about holomorphic functions we refer the reader to the classical [11]. We begin by introducing some notations.

\subsection{General terminology}

Denote the coordinates in $\mathbb{C}^{n}$ by $z=\left(z_{1}, \ldots, z_{n}\right)$, with $z_{i}=x_{i}+\sqrt{-1} y_{i}$, where $x_{i}=\operatorname{Re}\left(z_{i}\right)$ and $y_{i}=\operatorname{Im}\left(z_{i}\right)$ are respectively the real and the imaginary parts of $z_{i}$. Consider the usual conjugation $\sigma: \mathbb{C}^{n} \rightarrow \mathbb{C}^{n}, z \mapsto \bar{z}=\left(\overline{z_{1}}, \ldots, \overline{z_{n}}\right)$, whose set of fixed points is $\mathbb{R}^{n}$. A subset $A \subset \mathbb{C}^{n}$ is $\sigma$-invariant if $\sigma(A)=A$; obviously, $A \cap \sigma(A)$ is the biggest $\sigma$-invariant subset of $A$. Let $\Omega \subset \mathbb{C}^{n}$ be a $\sigma$-invariant open set and let $F: \Omega \rightarrow \mathbb{C}$ be a holomorphic function. We say that $F$ is $\sigma$-invariant if $F(z)=\overline{(F \circ \sigma)(z)}$ for all $z \in \Omega$. This implies that $F$ restricts to a (real) analytic function on $\Omega \cap \mathbb{R}^{n}$. In general, we denote by:

$$
\Re(F): \Omega \rightarrow \mathbb{C}, z \mapsto \frac{F(z)+\overline{(F \circ \sigma)(z)}}{2} \quad \text { and } \quad \Im(F): \Omega \rightarrow \mathbb{C}, z \mapsto \frac{F(z)-\overline{(F \circ \sigma)(z)}}{2 \sqrt{-1}}
$$


the real and the imaginary parts of $F$, which satisfy $F=\mathfrak{R}(F)+\sqrt{-1} \Im(F)$. Note that both are $\sigma$-invariant holomorphic functions. We will use the standard notation $\mathcal{O}_{\Omega}$ (respectively $\mathcal{O}_{\mathbb{R}^{n}}$ ) for the sheaf of holomorphic function germs (respectively of real analytic function germs). For the sake of clearness, the ring of holomorphic functions on $\Omega$ is denoted by $\mathcal{H}(\Omega)$ while the ring of analytic functions on $\mathbb{R}^{n}$ is denoted by $\mathcal{O}\left(\mathbb{R}^{n}\right)$. Given a holomorphic function $F \in \mathcal{H}(\Omega)$, we denote $\{F=$ $0\}=\{z \in \Omega: F(z)=0\}$; analogously, $\{f=0\}$ denotes the zeroset $\{x \in W$ : $f(x)=0\}$ of an analytic function $f \in \mathcal{O}(W)$ on an open subset $W \subset \mathbb{R}^{n}$. The notations Int and $\mathrm{Cl}$ stand for topological interiors and closures, respectively.

\subsection{Globally principal ideals}

We are mainly concerned with $X=\mathbb{R}^{n}$ or a contractible open Stein subsets $\Omega$ of $\mathbb{C}^{n}$ because on this type spaces each locally principal analytic (coherent) sheaf of ideals $\mathcal{F}$ is globally principal. As a classical application of Cartan's Theorem B (see [7]), this kind of sheaves provides a global section of the sheaf $\mathcal{D}=\mathcal{O}_{X} / \mathcal{O}_{X}^{*}$ of germs of positive divisors on $X$. By means of the long exact sequence of cohomology, we deduce that $\mathcal{F}$ has a global generator $f \in H^{0}\left(X, \mathcal{O}_{X}\right)$ if $H^{1}\left(X, \mathcal{O}_{X}^{*}\right)=0$. Using the exponential standard exact sequences of sheaves corresponding to $X$, we deduce that

$$
H^{1}\left(X, \mathcal{O}_{X}^{*}\right) \cong\left\{\begin{array}{l}
H^{1}\left(\mathbb{R}^{n}, \mathbb{Z}_{2}\right)=0 \text { if } X=\mathbb{R}^{n}, \\
H^{2}(\Omega, \mathbb{Z})=0 \text { if } X=\Omega .
\end{array}\right.
$$

In what follow we will use freely this fact: For such kind of analytic spaces $X$, each locally principal analytic (coherent) sheaf of ideals $\mathcal{F}$ on $X$ is globally principal. Of course, two global generators of $\mathcal{F}$ differ just by a unit. Recall also, the following fact that will be used freely: Given an open neighborhood $\Omega_{0}$ of $\mathbb{R}^{n}$ in $\mathbb{C}^{n}$, there exists a $\sigma$-invariant contractible open Stein neighborhood $\Omega$ of $\mathbb{R}^{n}$ in $\mathbb{C}^{n}$ contained in $\Omega_{0}$ (see [7, Section 1, Proposition 1]).

\subsection{Real and complex sheaf-products}

For our purposes, given a (countable) collection of either analytic functions on $\mathbb{R}^{n}$ or holomorphic functions on a contractible open Stein subsets $\Omega$ of $\mathbb{C}^{n}$ whose zero sets constitute a locally finite family, we need to construct an either analytic or holomorphic function which behaves (up to multiplication by a unit) as the (possibly infinite) product of all the functions in the collection. To that end, we recall a wellknown procedure that will be called sheaf-product for short. More precisely,

Definition 2.1. Let $\left\{f_{k}\right\}_{k \geq 1}$ be a collection of analytic functions on $\mathbb{R}^{n}$ such that the family $\left\{X_{k}=\left\{f_{k}=0\right\}\right\}_{k \geq 1}$ of their zero sets is locally finite in $\mathbb{R}^{n}$. Then, there exists a unique global generator $f \in \mathcal{O}\left(\mathbb{R}^{n}\right)$ (up to multiplication by a unit) of the locally principal analytic sheaf of ideals

$$
\mathcal{F}_{x}= \begin{cases}\prod_{k \geq 1, x \in X_{k}} f_{k, x} \mathcal{O}_{\mathbb{R}_{x}^{n}} & \text { if } x \in X=\bigcup_{k \geq 1} X_{k}, \\ \mathcal{O}_{\mathbb{R}^{n}, x} & \text { if } x \in \mathbb{R}^{n} \backslash X .\end{cases}
$$


We will refer to this generator $f$ as the sheaf-product $\pi f_{k}$ of the analytic functions $f_{k}$. Notice that if each $f_{k}$ is positive semidefinite, we may always assume that so is $\pi f_{k}$.

Analogously, given a collection $\left\{F_{k}\right\}_{k \geq 1}$ of holomorphic functions on a contractible open Stein subset $\Omega$ of $\mathbb{C}^{n}$ such that the family $\left\{Z_{k}=\left\{F_{k}=0\right\}\right\}_{k \geq 1}$ of their zero sets is locally finite in $\Omega$, we define the sheaf-product $\pi F_{k}$ of the holomorphic functions $F_{k}$. Notice that, by means of the following lemma, if $\Omega \cap \mathbb{R}^{n} \neq \varnothing$ and $\Omega$ and each $F_{k}$ are $\sigma$-invariant, we may always choose $\pi F_{k}$ also $\sigma$-invariant.

Lemma 2.2. Let $\Omega$ be a $\sigma$-invariant contractible open subset of $\mathbb{C}^{n}$ such that $\Omega \cap$ $\mathbb{R}^{n} \neq \varnothing$ and let $F: \Omega \rightarrow \mathbb{C}$ be a holomorphic function. Suppose that there exists a unit $u \in \mathcal{H}(\Omega)$ such that $\overline{F \circ \sigma}=u F$. Then, there exists a unit $v \in \mathcal{H}(\Omega)$ such that $v F: \Omega \rightarrow \mathbb{C}$ is $\sigma$-invariant.

Proof. We may assume that $F$ is not identically zero. Since $\overline{F \circ \sigma}=u F$, we also have $F=\overline{u \circ \sigma} \overline{F \circ \sigma}$, and so, $\overline{F \circ \sigma}=u \overline{u \circ \sigma} \overline{F \circ \sigma}$; hence, $u \overline{u \circ \sigma}=1$. Since $\Omega$ is contractible, there exists a unit $v \in \mathcal{H}(\Omega)$ such that $v^{2}=u$. Notice that $v \overline{v \circ \sigma}=1$, because $(v \overline{v \circ \sigma})^{2}=u \overline{u \circ \sigma}=1$ and its restriction to $\Omega \cap \mathbb{R}^{n} \neq \varnothing$ is positive. Then,

$$
\overline{(v F) \circ \sigma}=\overline{v \circ \sigma} \overline{F \circ \sigma}=v(\overline{v \circ \sigma})^{2} \overline{F \circ \sigma}=v \overline{u \circ \sigma} \overline{F \circ \sigma}=v F,
$$

and so, $v F$ is $\sigma$-invariant, as wanted.

\subsection{Real and complex sheaf-extension}

Let $X \subset \mathbb{R}^{n}$ be a global analytic set and let $U$ be a neighborhood of $X$ in $\mathbb{R}^{n}$. Given an analytic function $g: U \rightarrow \mathbb{R}$ such that $\{g=0\} \subset X$, we would like to find a global analytic function $f: \mathbb{R}^{n} \rightarrow \mathbb{R}$ which behaves as an extension of $g$ (up to multiplication by a unit). We also approach the analogous situation in the complex case.

Definition 2.3. Let $X \subset \mathbb{R}^{n}$ be a global analytic set and let $f: W \rightarrow \mathbb{R}$ be an analytic function on a neighborhood $W$ of $X$ in $\mathbb{R}^{n}$ such that $\{f=0\} \subset X$. Then, there is a unique global generator $\widehat{f} \in \mathcal{O}\left(\mathbb{R}^{n}\right)$ (up to multiplication by a unit) of the locally principal analytic sheaf of ideals

$$
\mathcal{F}_{x}= \begin{cases}f_{x} \mathcal{O}_{\mathbb{R}^{n}, x} & \text { if } x \in X, \\ \mathcal{O}_{\mathbb{R}^{n}, x} & \text { if } x \in \mathbb{R}^{n} \backslash X .\end{cases}
$$

We call $\widehat{f}$ the sheaf-extension of $f$ to $\mathbb{R}^{n}$. Of course, if $f$ is positive semidefinite, we may assume that so is $\widehat{f}$.

Analogously, given a contractible open Stein subset $\Omega$ of $\mathbb{C}^{n}$, an analytic subset $Z$ of $\Omega$, an open neighborhood $U$ of $Z$ in $\Omega$ and a holomorphic function $F: U \rightarrow \mathbb{C}$ such that $\{F=0\} \subset Z$, we define the sheaf-extension $F$ of $F$ to $\Omega$. As before if $\Omega \cap \mathbb{R}^{n} \neq \varnothing$ and $\Omega, U$ and $F$ are $\sigma$-invariant, we may always choose $F$ also $\sigma$-invariant. 
For our purposes, it will be crucial to "sheaf-extend" holomorphically to a suitable common domain countable families of either analytic or holomorphic functions whose zero sets constitute a locally finite family. The following "topological" preliminary results will help us to achieve our aim.

Lemma 2.4. Let $X$ be a paracompact second countable topological space and let $\left\{T_{k}\right\}_{k \geq 1}$ be a locally finite family of closed sets in $X$. For each $k \geq 1$, let $V_{k}$ be an open neighborhood of $T_{k}$ in $X$. Then, there exists open neighborhoods $U_{k} \subset V_{k}$ of $T_{k}$ in $X$ such that the family $\left\{U_{k}\right\}_{k \geq 1}$ is locally finite in $X$.

Proof. For each $x \in X$ there is an open neighborhood $W^{x}$ of $x$ that meets only finitely many $T_{k}$. The family $\left\{W^{x}\right\}_{x \in X}$ is an open covering of $X$; hence, it has an open refinement $\left\{W_{\ell}\right\}_{\ell>1}$ which is countable and locally finite in $X$. We define $U_{k}^{\prime}=\bigcup_{W_{\ell} \cap T_{k} \neq \varnothing} W_{\ell}$ and observe that $T_{k} \subset U_{k}^{\prime}$. We claim that the family $\left\{U_{k}^{\prime}\right\}_{k \geq 1}$ is locally finite in $X$.

Indeed, fix a point $x \in X$ and let $V^{x}$ be a neighborhood of $x$ which intersects finitely many $W_{\ell}$, say $W_{\ell_{1}}, \ldots, W_{\ell_{r}}$. Now, the union $\bigcup_{j=1}^{r} W_{\ell_{j}}$ meets only finitely many $T_{k}$, say $T_{k_{1}}, \ldots, T_{k_{s}}$; hence, if $k \neq k_{1}, \ldots, k_{s}$, the intersection $U_{k}^{\prime} \cap V^{x}=\varnothing$. To finish, just take $U_{k}=U_{k}^{\prime} \cap V_{k}$.

Lemma 2.5. Let $\left\{\Omega_{k}\right\}_{k \geq 1}$ be a locally finite family of open subsets of $\mathbb{C}^{n}$ and for each $k \geq 1$ let $T_{k} \subset \Omega_{k}$ be a closed subset of $\Omega_{k}$ such that $\mathrm{Cl}_{\mathbb{C}^{n}}\left(T_{k}\right) \cap \mathbb{R}^{n}=T_{k} \cap \mathbb{R}^{n}$. Then, there exists a ( $\sigma$-invariant contractible Stein) open neighborhood $\Omega$ of $\mathbb{R}^{n}$ in $\mathbb{C}^{n}$ such that $\Omega \cap T_{k}$ is a closed subset of $\Omega$ for all $k \geq 1$.

Proof. The obstruction to take $\Omega_{0}=\bigcup_{k>1} \Omega_{k}$ as the open neighborhood of $\mathbb{R}^{n}$ in $\mathbb{C}^{n}$ in the statement appears essentially at the sets $\operatorname{Cl}_{\mathbb{C}^{n}}\left(T_{i}\right) \cap \Omega_{j}$ for $j \neq i$. To get rid of such obstruction, we proceed as follows.

First, since the family $\left\{\Omega_{k}\right\}_{k \geq 1}$ is locally finite in $\mathbb{C}^{n}$, so are the families $\left\{T_{k}\right\}_{k \geq 1},\left\{\mathrm{Cl}_{\mathbb{C}^{n}}\left(T_{k}\right)\right\}_{k \geq 1}$ and $\left\{\mathrm{Cl}_{\mathbb{C}^{n}}\left(\bar{T}_{k}\right) \backslash \Omega_{k}\right\}_{k \geq 1}$. Thus,

$$
S=\bigcup_{k \geq 1} \mathrm{Cl}_{\mathbb{C}^{n}}\left(T_{k}\right) \quad \text { and } \quad C_{k}=\bigcup_{j \neq k} \mathrm{Cl}_{\mathbb{C}^{n}}\left(T_{j}\right) \backslash \Omega_{j}
$$

are closed subset of $\mathbb{C}^{n}$. Consider the open set of $\mathbb{C}^{n}$

$$
\Omega=\left(\mathbb{C}^{n} \backslash S\right) \cup \bigcup_{k \geq 1} \Omega_{k} \backslash C_{k} .
$$

We check first that $\mathbb{R}^{n} \subset \Omega$. Since $\mathrm{Cl}_{\mathbb{C}^{n}}\left(T_{k}\right) \cap \mathbb{R}^{n}=T_{k} \cap \mathbb{R}^{n}$, we have

$$
C_{k} \cap \mathbb{R}^{n}=\bigcup_{j \neq k}\left(\mathrm{Cl}_{\mathbb{C}^{n}}\left(T_{j}\right) \cap \mathbb{R}^{n}\right) \backslash \Omega_{j}=\bigcup_{j \neq k}\left(T_{j} \cap \mathbb{R}^{n}\right) \backslash \Omega_{j}=\varnothing,
$$

for all $k \geq 1$ and $S \cap \mathbb{R}^{n}=\bigcup_{k \geq 1} T_{k} \cap \mathbb{R}^{n}$. Thus, since $T_{k} \subset \Omega_{k}$,

$$
\mathbb{R}^{n} \cap \Omega=\left(\mathbb{R}^{n} \backslash \bigcup_{k \geq 1} T_{k} \cap \mathbb{R}^{n}\right) \cup \bigcup_{k \geq 1} \Omega_{k} \cap \mathbb{R}^{n}=\mathbb{R}^{n} .
$$


Next, we show that each intersection $T_{\ell} \cap \Omega$ is closed in $\Omega$ for $\ell \geq 1$. Fix $\ell \geq 1$ and observe that since $\Omega$ is open in $\mathbb{C}^{n}$, we have $\mathrm{Cl}_{\Omega}\left(T_{\ell} \cap \Omega\right)=\mathrm{Cl}_{\mathbb{C}^{n}}\left(T_{\ell}\right) \cap \Omega$; hence, it is enough to show that $\mathrm{Cl}_{\mathbb{C}^{n}}\left(T_{\ell}\right) \cap \Omega \subset T_{\ell}$ for each $\ell \geq 1$. Indeed, since $T_{\ell}$ is closed in $\Omega_{\ell}$, we have $\mathrm{Cl}_{\mathbb{C}^{n}}\left(T_{\ell}\right) \cap \Omega_{\ell}=T_{\ell}$; hence, since $\mathrm{Cl}_{\mathbb{C}^{n}}\left(T_{\ell}\right) \backslash \Omega_{\ell} \subset C_{k}$ if $k \neq \ell$, we deduce

$$
\begin{aligned}
\mathrm{Cl}_{\mathbb{C}^{n}}\left(T_{\ell}\right) \cap \Omega=\bigcup_{k \geq 1} \mathrm{Cl}_{\mathbb{C}^{n}}\left(T_{\ell}\right) \cap\left(\Omega_{k} \backslash C_{k}\right) \\
\mathrm{Cl}_{\mathbb{C}^{n}}\left(T_{\ell}\right) \cap \Omega_{\ell} \cup \bigcup_{k \neq \ell} \mathrm{Cl}_{\mathbb{C}^{n}}\left(T_{\ell}\right) \backslash\left(\mathrm{Cl}_{\mathbb{C}^{n}}\left(T_{\ell}\right) \backslash \Omega_{\ell}\right)=T_{\ell},
\end{aligned}
$$

as wanted. As always, we may assume that $\Omega$ is $\sigma$-invariant contractible and Stein.

Lemma 2.6. Let $V$ be an open neighborhood of $\mathbb{R}^{n}$ in $\mathbb{C}^{n}$ and let $Z \subset V$ be a closed subset of $V$. Let $\Omega$ be an open subset of $\mathbb{C}^{n}$ such that $Z \cap \mathbb{R}^{n} \subset \Omega \subset V$. Then, $\mathrm{Cl}_{\mathbb{C}^{n}}(Z \cap \Omega) \cap \mathbb{R}^{n}=(Z \cap \Omega) \cap \mathbb{R}^{n}$.

Proof. Since $Z$ is closed in $V$, we have $\operatorname{Cl}_{\mathbb{C}^{n}}(Z) \cap V=Z$. Thus, since $\mathbb{R}^{n} \subset V$, we have $\mathrm{Cl}_{\mathbb{C}^{n}}(Z) \cap \mathbb{R}^{n}=\mathrm{Cl}_{\mathbb{C}^{n}}(Z) \cap V \cap \mathbb{R}^{n}=Z \cap \mathbb{R}^{n}$; hence,

$$
Z \cap \mathbb{R}^{n} \subset \mathrm{Cl}_{\mathbb{C}^{n}}(Z \cap \Omega) \cap \mathbb{R}^{n} \subset \mathrm{Cl}_{\mathbb{C}^{n}}(Z) \cap \mathbb{R}^{n}=Z \cap \Omega \cap \mathbb{R}^{n},
$$

and we are done.

Next, we point out here a sufficient and necessary condition to assure that a countable family of closed subsets of an open set $\Omega \subset \mathbb{C}^{n}$ is locally finite.

Lemma 2.7. Let $\Omega \subset \mathbb{C}^{n}$ be an open set and let $\left\{T_{\ell}\right\}_{\ell \geq 1}$ be a family of closed subsets of $\Omega$. Then, the family $\left\{T_{\ell}\right\}_{\ell \geq 1}$ is locally finite if and only if there exists an exhaustion $\left\{K_{\ell}\right\}_{\ell \geq 1}$ of $\Omega$ by compact sets such that $T_{\ell} \cap K_{\ell}=\varnothing$ for all $\ell \geq 1$.

Proof. The if part is straightforward and we do not include the concrete details. For the converse, we begin by fixing an exhaustion $\left\{L_{k}\right\}_{k \geq 1}$ of $\Omega$ by compact sets, that is, $\operatorname{Int}_{\Omega}\left(L_{1}\right) \neq \varnothing, L_{k} \subset \operatorname{Int}_{\Omega}\left(L_{k+1}\right)$ and $\Omega=\bigcup_{k \geq 1} L_{k}$. For each $k \geq 1$, we define

$$
\ell(k)=\sup \left\{\ell \geq 1: L_{k} \cap T_{\ell} \neq \varnothing\right\} .
$$

and notice that $\ell(k)<+\infty$ because the family $\left\{T_{\ell}\right\}_{\ell \geq 1}$ is locally finite. Morever, the sequence $\{\ell(k)\}_{k \geq 1}$ is increasing and it tends to $+\infty$ because $\Omega=\bigcup_{k \geq 1} L_{k}$. Next, we choose a closed ball $L_{0}$ contained in $\operatorname{Int}_{\Omega}\left(L_{1}\right) \backslash \bigcup_{j=1}^{\ell(1)} T_{j}$ and define

$$
K_{\ell}^{\prime}= \begin{cases}L_{0} & \text { if } \ell(0)=1 \leq \ell \leq \ell(1) \\ L_{k} & \text { if } \ell(k)+1 \leq \ell \leq \ell(k+1)\end{cases}
$$

Observe that $K_{\ell}^{\prime} \cap T_{\ell}=\varnothing, K_{\ell(k)}^{\prime} \subset \operatorname{Int}_{\Omega}\left(K_{\ell(k)+1}^{\prime}\right)$ for all $k \geq 0$ and $\bigcup_{\ell \geq 1} K_{\ell}^{\prime}=\Omega$. Finally, modifying slightly the compact sets $K_{\ell}^{\prime}$, we obtain the desired exhaustion $\left\{K_{\ell}\right\}_{\ell \geq 1}$. 


\subsection{Sheaf-extensions to a suitable common domain}

After the previous preparation, we approach the holomorphic "sheaf-extension" to a suitable common (complex) domain of a collection of either analytic functions on $\mathbb{R}^{n}$ whose zero sets constitute a locally finite family of $\mathbb{R}^{n}$ or holomorphic functions whose (complex) domains of definition constitute a locally finite family of open subsets of $\mathbb{C}^{n}$. Namely,

Lemma 2.8. Let $\left\{f_{k}\right\}_{k \geq 1}$ be a collection of analytic functions on $\mathbb{R}^{n}$ such that the family $\left\{X_{k}=\left\{f_{k}=0\right\}\right\}_{k \geq 1}$ of their zero sets is locally finite in $\mathbb{R}^{n}$. Then, there exist a $\sigma$-invariant open neighborhood $\Omega$ of $\mathbb{R}^{n}$ in $\mathbb{C}^{n}$ and $\sigma$-invariant holomorphic functions $\widehat{F}_{k} \in \mathcal{H}(\Omega)$ such that the family $\left\{\left\{\widehat{F}_{k}=0\right\}\right\}_{k \geq 1}$ of their zero sets is locally finite in $\mathbb{C}^{n}$ and for each $k \geq 1$ there is a positive unit $u_{k} \in \mathcal{O}\left(\mathbb{R}^{n}\right)$ satisfying $\left.\widehat{F}_{k}\right|_{\mathbb{R}^{n}}=u_{k} f_{k}$.

Proof. We may assume that all the $f_{k}$ are non identically zero. For each $k \geq 1$, let $V_{k} \subset \mathbb{C}^{n}$ be a $\sigma$-invariant open neighborhood of $\mathbb{R}^{n}$ in $\mathbb{C}^{n}$ such that $f_{k}$ extends to a $\sigma$-invariant holomorphic function $F_{k}: V_{k} \rightarrow \mathbb{C}$. By Lemma 2.4, there is a locally finite family in $\mathbb{C}^{n}$ of $\sigma$-invariant open sets $\left\{\Omega_{k}\right\}_{k \geq 1}$ such that $X_{k} \subset \Omega_{k} \subset$ $V_{k}$. Let $T_{k}=\left\{F_{k}=0\right\} \cap \Omega_{k}$ which is $\sigma$-invariant and, by Lemma 2.6, satisfy $\mathrm{Cl}_{\mathbb{C}^{n}}\left(T_{k}\right) \cap \mathbb{R}^{n}=X_{k}=T_{k} \cap \mathbb{R}^{n}$.

Next, by Lemma 2.5 , there is a $\sigma$-invariant open neighborhood $\Omega$ of $\mathbb{R}^{n}$ in $\mathbb{C}^{n}$ such that each intersection $\Omega \cap T_{k}$ is a closed subset of $\Omega$. Next, for each $k \geq 1$ let $\widehat{F}_{k} \in \mathcal{H}(\Omega)$ be a $\sigma$-invariant sheaf-extension of $F_{k}$ to $\Omega$. A straightforward computation shows that the holomorphic functions $\left\{\widehat{F}_{k}\right\}_{k \geq 1}$ satisfy the desired conditions.

Lemma 2.9. Let $\left\{\Omega_{k}\right\}_{k \geq 1}$ be a locally finite family in $\mathbb{C}^{n}$ of $\sigma$-invariant open sets and for each $k \geq 1$ let $G_{k}: \Omega_{k} \rightarrow \mathbb{C}$ be a $\sigma$-invariant holomorphic function. Denote $S_{k}=\left\{G_{k}=0\right\}$ and assume that $S_{k} \cap \mathbb{R}^{n}$ is a (closed) global analytic set of $\mathbb{R}^{n}$ and $\mathrm{Cl}_{\mathbb{C}^{n}}\left(S_{k}\right) \cap \mathbb{R}^{n}=S_{k} \cap \mathbb{R}^{n}$ for each $k \geq 1$. Then, there are a $\sigma$-invariant open neighborhood $\Omega$ of $\mathbb{R}^{n}$ in $\mathbb{C}^{n}$ such that $S_{k} \cap \Omega$ is closed in $\Omega$ and $\sigma$-invariant sheaf-extensions $\widehat{G}_{k} \in \mathcal{H}(\Omega)$ of the functions $G_{k}$.

Proof. We may assume that $G_{k}$ is not identically zero in any of the connected components of $\Omega_{k}$. Next, by Lemma 2.5 , there is a $\sigma$-invariant contractible open Stein neighborhood $\Omega$ of $\mathbb{R}^{n}$ in $\mathbb{C}^{n}$ such that $Z_{k}=S_{k} \cap \Omega$ is a closed subset of $\Omega$ for all $k \geq 1$. Next, for each $k \geq 1$ let $\widehat{G}_{k} \in \mathcal{H}(\Omega)$ be a $\sigma$-invariant sheaf-extension of $G_{k}$ to $\Omega$. A straightforward computation shows that the holomorphic functions $\left\{\widehat{G}_{k}\right\}_{k \geq 1}$ satisfy the desired conditions.

\subsection{Infinite sums of squares}

As we have already commented, we are concerned about infinite sum of squares and we recall here some statements from [2]. We begin with the precise definition of infinite sum of squares of analytic functions. 
Definition 2.10. Let $W \subset \mathbb{R}^{n}$ be an open set. An infinite sum of squares of analytic functions on $W$ is a series $\sum_{k \geq 1} f_{k}^{2}$ where all $f_{k} \in \mathcal{O}(W)$, such that:

(i) The $f_{k}$ 's have holomorphic extensions $F_{k}$ 's, all defined on the same open neighborhood $\Omega$ of $W$ in $\mathbb{C}^{n}$, and

(ii) $\sum_{k \geq 1} \sup _{K}\left|F_{k}\right|^{2}<+\infty$ for every compact set $K \subset \Omega$.

Accordingly, the infinite sum of squares $\sum_{k>1} f_{k}^{2}$ defines well an analytic function $f$ on $W=\Omega \cap \mathbb{R}^{n}$ and we write $f=\sum_{k>1} f_{k}^{2} \in \mathcal{O}(W)$. Of course, this definition trivially includes finite sums of squares. Hence, it makes sense to say that an element of the ring $\mathcal{O}(W)$ is a sum of $p$ squares in $\mathcal{O}(W)$, even for $p=+\infty$. Next, an analytic function $f: W \rightarrow \mathbb{R}$ is a sum of $p \leq+\infty$ squares (of meromorphic functions) on $W$ if there is $g \in \mathcal{O}(W) \backslash\{0\}$ such that $g^{2} f$ is a sum of $p$ squares of analytic functions on $W$. The set $\{g=0\}$ is the zeroset of the denominators of that representation of $f$ as a sum of squares. More generally, we say that an analytic function $f: W \rightarrow \mathbb{R}$ is a sum of squares (of meromorphic functions) at $\{f=0\}$ if there is an open neighborhood $V$ of $\{f=0\}$ in $W$ such that $\left.f\right|_{V}$ is a sum of squares on $V$.

The choice of a suitable sum of squares representation is a crucial matter and we need often to have controlled denominators, that is, the zeroset $\{g=0\}$ of the denominators of the representation of $f$ as sum of squares is contained in the zeroset $\{f=0\}$. In $[2,4.1]$ we proved the following.

Lemma 2.11. Let $W \subset \mathbb{R}^{n}$ be an open set and let $f: W \rightarrow \mathbb{R}$ be an analytic function which is a sum of $p \leq+\infty$ squares on $W$. Then, $f$ is a sum of $q \leq$ $2^{n-1} p$ squares in a perhaps smaller neighborhood $W^{\prime}$ of $\{f=0\}$ with controlled denominators.

Moreover, the "local" representations as sum of squares on a neighborhood of the zeroset of a positive semidefinite analytic function can be always extended to $\mathbb{R}^{n}$ by means of the following result proved in $[2,1.8,4.1]$. Although the statement we present here could seem more general than the one we presented there, its proof is exactly the same.

Theorem 2.12. Let $f: \mathbb{R}^{n} \rightarrow \mathbb{R}$ be an analytic function and suppose that $f$ is a sum of $p \leq+\infty$ squares at $\{f=0\}$. Then, there exist analytic functions $g, h, q: \mathbb{R}^{n} \rightarrow \mathbb{R}$ such that $q$ is a sum of $2^{n-1} p$ squares of analytic functions on $\mathbb{R}^{n},\{g=0\} \subset\{h=0\}=\{f=0\}$ and $g^{2} f=h^{2}+q$.

The main tool to prove the previous result in case $p=+\infty$ involves the following result (proved in $[2,2.4]$ ) which approaches the extension of sum of squares of $\sigma$-invariant holomorphic functions.

Proposition 2.13. Let $\Omega$ be a $\sigma$-invariant open Stein neighborhood of $\mathbb{R}^{n}$ in $\mathbb{C}^{n}$ and let $\Phi \in \mathcal{H}(\Omega)$ be a $\sigma$-invariant holomorphic function. Let $V$ be a $\sigma$-invariant nonempty open set such that $\{\Phi=0\} \cap V$ is closed in $\Omega$. Let $\left\{C_{k}\right\}_{k \geq 1} \subset \mathcal{H}(V)$ be 
a collection of $\sigma$-invariant holomorphic functions such that $\sum_{k} \sup _{L}\left|C_{k}\right|^{2}<+\infty$ for every compact set $L \subset V$. Then, there exist $\sigma$-invariant holomorphic functions $A_{k}: \Omega \rightarrow \mathbb{C}$, such that $\sum_{k} \sup _{K}\left|A_{k}\right|^{2}<+\infty$ for every compact set $K \subset \Omega$ and $\left.\Phi\right|_{V}$ divides all the differences $\left.A_{k}\right|_{V}-C_{k}$.

A relevant consequence of Subsection 2.5 and Proposition 2.13 is the following result which concerns the holomorphic extension of countably many sums of squares to the same complex neighborhood.

Lemma 2.14. Let $\left\{f_{k}\right\}_{k \geq 1}$ be a collection of analytic functions on $\mathbb{R}^{n}$ such that:

- the family $\left\{X_{k}=\left\{f_{k}=0\right\}\right\}_{k \geq 1}$ of their zero sets is locally finite in $\mathbb{R}^{n}$, and

- each $f_{k}$ is a sum of $p_{k}$ squares at $X_{k}$, where $1 \leq p_{k} \leq+\infty$.

Then, there are a $\sigma$-invariant open neighborhood $\Omega$ of $\mathbb{R}^{n}$ in $\mathbb{C}^{n}$, positive real constants $c_{k}$ for $k \geq 1$ and $\sigma$-invariant homolomorphic functions $G_{k}, \widehat{F}_{k}, A_{k j} \in$ $\mathcal{H}(\Omega)$ for $k \geq 1$ and $1 \leq j \leq q_{k}=2^{n+1} p_{k}$ such that:

(i) $\left.\widehat{F}_{k}\right|_{\mathbb{R}^{n}}=u_{k} f_{k}$ for a positive unit $u_{k} \in \mathcal{O}\left(\mathbb{R}^{n}\right)$.

(ii) The family $\left\{\left\{G_{k}^{2} \widehat{F}_{k}=0\right\}\right\}_{k \geq 1}$ is locally finite in $\Omega$ and $\left\{G_{k}^{2} \widehat{F}_{k}=0\right\} \cap \mathbb{R}^{n}=X_{k}$.

(iii) The sum $A_{k}=\left(d_{k} G_{k}^{2} \widehat{F}_{k}\right)^{2}+\sum_{j=1}^{q_{k}} A_{k j}^{2}$ converges in $\Omega$ in the sense of Definition 2.10(ii) for each family of real values $\left\{d_{k}\right\}_{k \geq 1}$ satisfying $d_{k} \geq c_{k}$. Moreover,

- $\left\{\left.A_{k}\right|_{\mathbb{R}^{n}}=0\right\}=X_{k}$ and the family $\left\{\left\{A_{k}=0\right\}\right\}_{k \geq 1}$ is locally finite in $\Omega$; and

- $A_{k}=G_{k}^{2} \widehat{F}_{k} Q_{k}$ for some $\sigma$-invariant $Q_{k} \in \mathcal{H}(\Omega)$, which does not vanish on a neighborhood $V_{k}$ of $\left\{G_{k}^{2} \widehat{F}_{k}=0\right\} \cup \mathbb{R}^{n}$.

Proof. We will split the proof into several steps.

STARTING POINT. By Theorem 2.12 we can find a representation of $f_{k}$ as a sum of $q_{k}=2^{n-1} p_{k}$ squares on $\mathbb{R}^{n}$ with controlled denominators. This provides:

(1) By Lemma 2.8 , there are a $\sigma$-invariant open neighborhood $\Omega_{0}$ of $\mathbb{R}^{n}$ in $\mathbb{C}^{n}$ and $\sigma$-invariant holomorphic functions $\widehat{F}_{k} \in \mathcal{H}\left(\Omega_{0}\right)$ such that the family $\left\{\left\{\widehat{F}_{k}=0\right\}\right\}_{k \geq 1}$ is locally finite in $\mathbb{C}^{n}, \widehat{F}_{k} \mid \mathbb{R}^{n}=u_{k} f_{k}$ and $u_{k} \in \mathcal{O}\left(\mathbb{R}^{n}\right)$ is a positive unit.

(2) There are $\sigma$-invariant open neighborhoods $U_{k}$ of $\mathbb{R}^{n}$ in $\mathbb{C}^{n}$ and $\sigma$-invariant holomorphic functions $G_{k}^{\prime}, B_{k j}: U_{k} \rightarrow \mathbb{C}$ such that $G_{k}^{\prime 2} \widehat{F}_{k}=\sum_{j=1}^{q_{k}} B_{k j}^{2}$ and $\left\{G_{k}^{\prime}=0\right\} \cap \mathbb{R}^{n} \subset X_{k}$.

STEP 1: EXTENSION OF DENOMinators. The family $\left\{T_{k}=\left\{\widehat{F}_{k}=0\right\} \cap U_{k}\right\}_{k \geq 1}$ is, by (1) above, locally finite in $\mathbb{C}^{n}$. Now, by Lemma 2.4 , there is a locally finite family in $\mathbb{C}^{n}$ of $\sigma$-invariant open sets $\left\{\Omega_{k}\right\}_{k \geq 1}$ in $\mathbb{C}^{n}$ such that $T_{k} \subset \Omega_{k} \subset U_{k}$. Consider the $\sigma$-invariant sets $S_{k}=\left\{\left.G_{k}^{\prime 2} \widehat{F}_{k}\right|_{U_{k}}=0\right\} \cap \Omega_{k}$. By Lemma 2.6, we have $\mathrm{Cl}_{\mathbb{C}^{n}}\left(S_{k}\right) \cap \mathbb{R}^{n}=X_{k}=S_{k} \cap \mathbb{R}^{n}$ for all $k \geq 1$.

Now, by Lemma 2.9, we find a $\sigma$-invariant open neighborhood $\Omega \subset \Omega_{0}$ of $\mathbb{R}^{n}$ in $\mathbb{C}^{n}$ such that $\Omega \cap S_{k}$ is a closed subset of $\Omega$ and $\sigma$-invariant sheaf-extensions 
$G_{k}=\widehat{G}_{k}^{\prime} \in \mathcal{H}(\Omega)$ of the functions $G_{k}^{\prime}$. To simplify notations, denote again by $\widehat{F}_{k}$ its restriction to $\Omega$ and by $\Omega_{k}$ and $S_{k}$ their respective intersections with $\Omega$.

Let $v_{k} \in \mathcal{H}\left(\Omega_{k}\right)$ be a $\sigma$-invariant unit such that $\left.G_{k}\right|_{\Omega_{k}}=\left.v_{k} G_{k}^{\prime}\right|_{\Omega_{k}}$. Thus, if we write $C_{k j}=\left.v_{k} B_{k j}\right|_{\Omega_{k}}$, we have

$$
\left.\left(G_{k}^{2} \widehat{F}_{k}\right)\right|_{\Omega_{k}}=\left.v_{k}^{2}\left(G_{k}^{\prime 2} \widehat{F}_{k}\right)\right|_{\Omega_{k}}=\sum_{j=1}^{q_{k}}\left(v_{k} B_{k j} \mid \Omega_{k}\right)^{2}=\sum_{j=1}^{q_{k}} C_{k j}^{2} .
$$

Moreover, the family $\left\{S_{k}=\left\{G_{k}^{2} \widehat{F}_{k}=0\right\}\right\}_{k \geq 1}$ is locally finite in $\Omega$ and $\left\{G_{k}^{2} \widehat{F}_{k}=\right.$ $0\} \cap \mathbb{R}^{n}=X_{k}$ for all $k \geq 1$.

STEP 2: GLOBALIZATION OF SUMS OF SQUARES. Here we replace the sums of squares $\sum_{j=1}^{q_{k}} C_{k j}^{2}$, which are defined only on the sets $\Omega_{k}$, by global sum of squares $\sum_{j=1}^{q_{k}} A_{k j}^{2}$ satisfying the conditions in the statement.

Fix $k \geq 1$ and observe that $\Omega_{k} \cap\left\{G_{k}^{2} \widehat{F}_{k}=0\right\}=S_{k}$ which is a closed subset of $\Omega$. We apply Proposition 2.13 to $\Phi=\left(G_{k}^{2} \widehat{F}_{k}\right)^{2}, V=\Omega_{k}$ and $C_{j}=C_{k j}$ and we find $\sigma$-invariant holomorphic functions $A_{k j}$ on $\Omega$, such that

- $\sum_{j=1}^{q_{k}} \sup _{K}\left|A_{k j}\right|^{2}<+\infty$ for all compact sets $K \subset \Omega$, and

- $\left(G_{k}^{2} \widehat{F}_{k}\right)^{2}$ divides $A_{k j}-C_{k j}$ on $\Omega_{k}$.

Next, let $\left\{K_{k}\right\}_{k \geq 1}$ be an exhaustion of $\Omega$ by compact sets such that $S_{k} \cap K_{k}=\varnothing$ for all $k \geq 1$ (see Lemma 2.7). Denote $A_{k}^{\prime}=\sum_{j=1}^{q_{k}} A_{k j}^{2}$ and let

$$
c_{k}=1+\frac{\max _{K_{k}}\left(\left|A_{k}^{\prime}\right|\right)}{\min _{K_{k}}\left(\left|G_{k}^{2} \widehat{F}_{k}\right|^{2}\right)}>0 .
$$

Write $A_{k 0}=d_{k} G_{k}^{2} \widehat{F}_{k}$ for $d_{k} \geq c_{k}$ and define $A_{k}=A_{k 0}^{2}+A_{k}^{\prime}=\sum_{j \geq 0} A_{k j}^{2}$. Each function $A_{k}$ is holomorphic and $\sigma$-invariant on $\Omega$ and its restriction to $\mathbb{R}^{n}$ vanishes only at $X_{k}$, because so does $A_{k 0} \mid \mathbb{R}^{n}$. Moreover, let us check that for such choice of $d_{k}$ 's the family $\left\{\left\{A_{k}=0\right\}\right\}_{k \geq 1}$ is locally finite in $\Omega$. By Lemma 2.7, it is enough to prove that $\left\{A_{k}=0\right\} \cap K_{k}=\varnothing$ for all $k \geq 1$.

Indeed, suppose by way of contradiction that there exists $z \in\left\{A_{k}=0\right\} \cap K_{\ell(k)}$; then, $\left(d_{k} G_{k}^{2} \widehat{F}_{k}\right)^{2}(z)=-A_{k}^{\prime}(z)$ and since $\left(G_{k}^{2} \widehat{F}_{k}\right)(z) \neq 0$, we have

$$
1 \leq c_{k}^{2} \leq d_{k}^{2}=\frac{\left|A_{k}^{\prime}(z)\right|}{\left|\left(G_{k}^{2} \widehat{F}_{k}\right)(z)\right|^{2}}<1+\frac{\max _{K_{\ell(k)}}\left(\left|A_{k}^{\prime}\right|\right)}{\min _{K_{\ell(k)}}\left(\left|G_{k}^{2} \widehat{F}_{k}\right|^{2}\right)}=c_{k} \leq d_{k},
$$

a contradiction.

Next, we claim that: The meromorphic function $Q_{k}=A_{k} /\left(G_{k}^{2} \widehat{F}_{k}\right)$ is holomorphic on $\Omega$ and it does not vanish on a neighborhood of $\left\{G_{k}^{2} \widehat{F}_{k}=0\right\} \cup \mathbb{R}^{n}$.

Since the zeroset of $G_{k}^{2} \widehat{F}_{k}$ in $\Omega$ is $S_{k} \subset \Omega_{k}$, we have

$$
\left\{A_{k}=0\right\} \cap \mathbb{R}^{n}=X_{k} \subset S_{k} \subset \Omega_{k}
$$


and our claim reduces to show that $Q_{k}$ is holomorphic unit on an open neighborhood of $S_{k}$. Indeed, on $\Omega_{k}$ we have:

$$
\sum_{j=1}^{q_{k}} A_{k j}^{2}-G_{k}^{2} \widehat{F}_{k}=\sum_{j=1}^{q_{k}} A_{k j}^{2}-\sum_{j=1}^{q_{k}} C_{k j}^{2}=\sum_{j=1}^{q_{k}}\left(A_{k j}^{2}-C_{k j}^{2}\right) .
$$

Of course, all involved series are convergent on the compact sets of $\Omega_{k}$. Also, by construction, $\left(G_{k}^{2} \widehat{F}_{k}\right)^{2}$ divides on $\Omega_{k}$ each term $A_{k j}^{2}-C_{k j}^{2}=\left(A_{k j}+C_{k j}\right)\left(A_{k j}-C_{k j}\right)$; hence, it divides their sum $\sum_{j=1}^{q_{k}} A_{k j}^{2}-G_{k}^{2} \widehat{F}_{k}$. Thus, we can write

$$
A_{k}=\sum_{j=0}^{q_{k}} A_{k j}^{2}=A_{k 0}^{2}+\sum_{j=1}^{q_{k}} A_{k j}^{2}=G_{k}^{2} \widehat{F}_{k}+H_{k}\left(G_{k}^{2} \widehat{F}_{k}\right)^{2}=Q_{k} G_{k}^{2} \widehat{F}_{k},
$$

where $H_{k} \in \mathcal{H}\left(\Omega_{k}\right)$ and $Q_{k}=1+H_{k} G_{k}^{2} \widehat{F}_{k}$ in $\Omega_{k}$; hence, shrinking $\Omega_{k}$ if necessary, we get that $Q_{k}$ is a $\sigma$-invariant holomorphic unit on $\Omega_{k}$, as wanted.

\section{Bounded analytic Pfister's formula}

The classical Pfister's formula (see [18]) says that in a field $K$ the subset of sums of $2^{r}$ squares is closed under multiplication. In order to ease the writing of what follows we will use the standard notation due to Pfister: Given an element $f$ of a ring $A$, the expression $f=p$ means that $f$ is a sum of $p$ squares in $A$; when several $p$ 's appear in the same formula, they need not be the same. For instance, Pfister's formula can be re-written as $2^{r} 2^{r}=2^{r}$. In fact, by means of complex numbers, quaternions and octonions we have also the equality $2^{r} 2^{r}=2^{r}$ for $r=1,2,3$ in any ring $A$. The main purpose of this section is to prove Theorems 1.1(ii) and 1.3. By means of Lemma 2.11, Theorem 1.1(ii) reduces to prove the following statement.

Theorem 3.1 (Bounded analytic Pfister's formula). Let $\left\{f_{k}\right\}_{k \geq 1}$ be a collection of analytic functions on $\mathbb{R}^{n}$ such that the family $\left\{X_{k}=\left\{f_{k}=0\right\}\right\}_{k \geq 1}$ of their zero sets is locally finite. Assume that each $f_{k}$ is a sum of $2^{r}$ squares at $X_{k}$ with controlled denominators (for a fixed $r$ ). Then, $\pi f_{k}$ is a sum of $2^{r+1}$ squares with controlled denominators.

\subsection{Pfister's multiplicative formulae}

One way to prove Pfister's multiplicative formulae consists in providing, given an element $a=2^{r}$ in $K$ (respectively $A$ ), a matrix $M_{a} \in \mathfrak{M}_{2^{r}}(K)$ of order $2^{r}$ and coefficients in $K$ (respectively in $A$ ) such that $M_{a}^{t} M_{a}=a I_{2^{r}}$. Once this is done, if $a, b$ are sums of $2^{r}$ squares, we have $\left(M_{a} M_{b}\right)^{t}\left(M_{a} M_{b}\right)=a b I_{2^{r}}$ and this provides a representation of $a b$ as a sum of $2^{r}$ squares; of course, $I_{2^{r}}$ denotes the 
identity matrix of the ring $\mathfrak{M}_{2^{r}}(K)$ (respectively $\mathfrak{M}_{2^{r}}(A)$ ). For our purposes, we present next a slight modification of the procedure to construct such matrices (see also $[16,7.1])$.

Lemma 3.2. Let $A$ be a unitary commutative ring and let $g_{i}, f_{i} \in A$ for $i=1,2$. Suppose that there exist matrices $M_{1}, M_{2} \in \mathfrak{M}_{2^{r}}(A)$ such that $M_{i}^{t} M_{i}=g_{i}^{2} f_{i} I_{2^{r}}$. Then, there is a matrix $M \in \mathfrak{M}_{2^{r+1}}(A)$ such that $M^{t} M=g^{2} f I_{2^{r}}$ where $g=$ $g_{1} g_{2}^{2} f_{2}$ and $f=f_{1}+f_{2}$.

Proof. A straighforward computation shows that

$$
M=\left(\begin{array}{cc}
g_{2}^{2} f_{2} M_{1} & -g_{1} g_{2} f_{2} M_{2} \\
g_{1} g_{2} f_{2} M_{2} & M_{2} M_{1}^{t} M_{2}
\end{array}\right) \in \mathfrak{M}_{2^{r}}(A)
$$

is the matrix we sought.

In our case $A=\mathcal{O}\left(\mathbb{R}^{n}\right)$, we are concerned about "controling denominators". This is done by means of the following result.

Lemma 3.3. Let $f, a \in \mathcal{O}\left(\mathbb{R}^{n}\right)$ be analytic functions such that $f=a^{2}+2^{r-1}$ in $\mathcal{O}\left(\mathbb{R}^{n}\right)$ and $\{a=0\}=\{f=0\}$. Then, there exist $g \in \mathcal{O}\left(\mathbb{R}^{n}\right)$ with $\{g=0\} \subset\{f=$ $0\}$ and a matrix $M \in \mathfrak{M}_{2^{r}}\left(\mathcal{O}\left(\mathbb{R}^{n}\right)\right)$ such that $M^{t} M=g^{2} f I_{2^{r}}$.

Proof. Indeed, if $r=1, f=a^{2}+b^{2}$ it is enough to take $g=1$ and

$$
M=\left(\begin{array}{cc}
a & -b \\
b & a
\end{array}\right) \in \mathfrak{M}_{2}\left(\mathcal{O}\left(\mathbb{R}^{n}\right)\right)
$$

Assume now $r>1$, and let $f_{1}=\left(\frac{3}{5} a\right)^{2}+2^{r-2}$ and $f_{2}=\left(\frac{4}{5} a\right)^{2}+2^{r-2}$ such that $f=f_{1}+f_{2}$. Notice that $\left\{f_{1}=0\right\}=\left\{f_{2}=0\right\}=\{a=0\}=\{f=0\}$. By induction, there are analytic functions $g_{1}, g_{2} \in \mathcal{O}\left(\mathbb{R}^{n}\right)$ such that $\left\{g_{i}=0\right\} \subset\left\{f_{i}=\right.$ $0\}$ and matrices $M_{i} \in \mathfrak{M}_{2^{r-1}}\left(\mathcal{O}\left(\mathbb{R}^{n}\right)\right)$ such that $M_{i}^{t} M_{i}=g_{i}^{2} f_{i} I_{2^{r-1}}$ for $i=1,2$. By Lemma 3.2, there is a matrix $M \in \mathfrak{M}_{2^{r+1}}\left(\mathcal{O}\left(\mathbb{R}^{n}\right)\right)$ such that $M^{t} M=g^{2} f I_{2^{r}}$ where $g=g_{1} g_{2}^{2} f_{2}$. Observe that $\{g=0\}=\left\{g_{1}=0\right\} \cup\left\{g_{2}=0\right\} \cup\left\{f_{2}=0\right\}=\{f=0\}$, as wanted.

Remark 3.4. (i) Of course, if $f=p$ in $\mathcal{O}\left(\mathbb{R}^{n}\right)$, there exists $a \in \mathcal{O}\left(\mathbb{R}^{n}\right)$ such that $f=a^{2}+p$ in $\mathcal{O}\left(\mathbb{R}^{n}\right)$ and $\{a=0\}=\{f=0\}$.

Indeed, $1+f$ is a positive unit of $\mathcal{O}\left(\mathbb{R}^{n}\right)$ and so $u=\frac{1}{\sqrt{1+f}} \in \mathcal{O}\left(\mathbb{R}^{n}\right)$. Thus, if we denote $a=f u$, we have $f=\left(f+f^{2}\right) u^{2}=a^{2}+u^{2} p=a^{2}+p$ and $\{a=0\}=\{f=0\}$. 
(ii) If $f=2^{r}$ in $\mathcal{O}\left(\mathbb{R}^{n}\right)$ with $r \leq 3$, we may choose a matrix $M_{f} \in \mathfrak{M}_{2^{r}}\left(\mathcal{O}\left(\mathbb{R}^{n}\right)\right)$ such that $M_{f}^{t} M_{f}=f I_{2^{r}}$. Namely,

$$
\begin{gathered}
f=a_{1}^{2}+a_{2}^{2}, \quad f=a_{1}^{2}+a_{2}^{2}+a_{3}^{2}+a_{4}^{2}, \quad f=a_{1}^{2}+a_{2}^{2}+a_{3}^{2}+a_{4}^{2}+a_{5}^{2}+a_{6}^{2}+a_{7}^{2}+a_{8}^{2} . \\
M_{f}=\left(\begin{array}{cc}
a_{1}-a_{2} \\
a_{2} & a_{1}
\end{array}\right), M_{f}=\left(\begin{array}{cccc}
a_{1}-a_{2}-a_{3}-a_{4} \\
a_{2} & a_{1} & a_{4} & -a_{3} \\
a_{3}-a_{4} & a_{1} & a_{2} \\
a_{4} & a_{3} & -a_{2} & a_{1}
\end{array}\right), M_{f}=\left(\begin{array}{cccccccc}
a_{1}-a_{2}-a_{3}-a_{4}-a_{5}-a_{6}-a_{7}-a_{8} \\
a_{2} & a_{1} & -a_{5}-a_{8} & a_{3} & -a_{7} & a_{6} & a_{4} \\
a_{3} & a_{5} & a_{1} & -a_{6}-a_{2} & a_{4} & -a_{8} & a_{7} \\
a_{4} & a_{8} & a_{6} & a_{1} & -a_{7} & -a_{3} & a_{5} & -a_{2} \\
a_{5}-a_{3} & a_{2} & a_{7} & a_{1} & -a_{8} & -a_{4} & a_{6} \\
a_{6} & a_{7} & -a_{4} & a_{3} & a_{8} & a_{1} & -a_{2} & -a_{5} \\
a_{7}-a_{6} & a_{8} & -a_{5} & a_{4} & a_{2} & a_{1} & -a_{3} \\
a_{8}-a_{4}-a_{7} & a_{2} & -a_{6} & a_{5} & a_{3} & a_{1}
\end{array}\right) .
\end{gathered}
$$

\subsection{Pfister's bundles}

Following some ideas developed in [13, Section 2] to approach the representation of a positive semidefinite analytic function on an analytic surface as sum of squares of analytic functions, we will associate a suitable analytic vector bundle endowed with a Riemannian metric to each finite sum of squares in $\mathcal{O}\left(\mathbb{R}^{n}\right)$.

Definition 3.5. Let $f \in \mathcal{O}\left(\mathbb{R}^{n}\right)$ be an analytic function. We define a Pfister's bundle for $f$ as a tuple $\mathscr{P}(f)=\left(E(f), \pi, \mathbb{R}^{n} ;\langle\cdot, \cdot\rangle, s\right)$ consisting of:

- an analytic vector bundle $\left(E(f), \pi, \mathbb{R}^{n}\right)$ of finite rank,

- a Riemannian metric $\langle\cdot, \cdot\rangle$ on $E$, and

- a global analytic section $s: \mathbb{R}^{n} \rightarrow E(f)$ such that $\langle s, s\rangle=f$.

Remark 3.6. An analytic vector bundle over $\mathbb{R}^{n}$ is topologically trivial because $\mathbb{R}^{n}$ is contractible; hence, it is, by [10], also analytically trivial. Thus, a Pfister's bundle $\mathscr{P}(f)$ for $f$ of rank $p$ admits $p$ unitary analytic sections $s_{1}, \ldots, s_{p}: \mathbb{R}^{n} \rightarrow E$ which are pairwise orthogonal, that is, $\left\langle s_{i}, s_{j}\right\rangle=\delta_{i j}$ for $1 \leq i, j \leq n$.

Next, we characterize the finite sums of squares in terms of Pfister's bundles. Namely:

Lemma 3.7. Let $f \in \mathcal{O}\left(\mathbb{R}^{n}\right)$ be an analytic function. Then, $f$ is a finite sum of squares if and only if there exist $g \in \mathcal{O}\left(\mathbb{R}^{n}\right) \backslash\{0\}$ and a Pfister's bundle $\mathscr{P}\left(g^{2} f\right)$ of finite rank for $g^{2} f$. Moreover, if $\mathscr{P}\left(g^{2} f\right)$ has rank $p$, then $f$ can be represented as a sum of $p$ squares and the zeroset of the denominators of such representation is contained in $\{g=0\}$.

Proof. Suppose first that $f$ is a sum of $2^{r-1}$ squares, that is, there is $g_{0} \in \mathcal{O}\left(\mathbb{R}^{n}\right) \backslash$ $\{0\}$ such that $g_{0}^{2} f$ is a sum of $2^{r-1}$ squares in $\mathcal{O}\left(\mathbb{R}^{n}\right)$. By Remark 3.4(i) and Lemma 3.3, there exist $g_{1} \in \mathcal{O}\left(\mathbb{R}^{n}\right) \backslash\{0\}$ and a matrix $M_{h} \in \mathfrak{M}_{2^{r}}\left(\mathcal{O}\left(\mathbb{R}^{n}\right)\right)$ such that $M_{h}^{t} M_{h}=h I_{2^{r}}$ where $h=\left(g_{0} g_{1}\right)^{2} f$; denote $g=g_{0} g_{1}$.

Write $p=2^{r}$ and consider the analytic vector bundle $\left(E, \pi, \mathbb{R}^{n}\right)$ where $E=$ $\mathbb{R}^{n} \times \mathbb{R}^{p}$ and $\pi: \mathbb{R}^{n} \times \mathbb{R}^{p} \rightarrow \mathbb{R}^{n}$ is the projection onto the first space. Consider 
the analytic Riemannian metric on $\left(E \oplus E, \pi^{\prime}, \mathbb{R}^{n}\right)=\left(\mathbb{R}^{n} \times \mathbb{R}^{2 p}, \pi^{\prime}\right)$ given by

$$
\langle\cdot, \cdot\rangle\left(x,\left(v_{1}, \ldots, v_{p}\right),\left(w_{1}, \ldots, w_{p}\right)\right)=\sum_{\ell=1}^{p} v_{\ell} w_{\ell} .
$$

Let $u \in \mathbb{R}^{p}$ be any unitary vector and consider the global section

$$
s: \mathbb{R}^{n} \rightarrow E, x \mapsto\left(x, M_{h}(x)(u)\right)
$$

Since $\langle s, s\rangle=h$, the tuple $\mathscr{P}(h)=\left(\left(E, \pi, \mathbb{R}^{n}\right), s\right)$ is a Pfister's bundle for $h=g^{2} f$.

Conversely, suppose that there exists a Pfister's bundle $\mathscr{P}\left(g^{2} f\right)=$ $\left(\left(E, \pi, \mathbb{R}^{n}\right),\langle\cdot, \cdot\rangle, s\right)$ of rank $p$ for $g^{2} f$, where $g \in \mathcal{O}\left(\mathbb{R}^{n}\right) \backslash\{0\}$. By Remark 3.6, there exists a family of analytic sections $\left\{s_{1}, \ldots, s_{p}\right\}$ of $\left(E, \pi, \mathbb{R}^{n}\right)$ such that $\left\langle s_{i}, s_{j}\right\rangle=\delta_{i j}$ for $1 \leq i, j \leq p$. Write now $s=\sum_{i=1}^{p} a_{i} s_{i}$, where $a_{i}=\left\langle s, s_{i}\right\rangle \in$ $\mathcal{O}\left(\mathbb{R}^{n}\right)$, and notice that

$$
g^{2} f=\langle s, s\rangle=a_{1}^{2}+\cdots+a_{p}^{2}
$$

is a sum of $p$ squares in $\mathcal{O}\left(\mathbb{R}^{n}\right)$; hence, $f$ is a sum of $p$ squares and the zeroset of the denominators of such representation is contained in $\{g=0\}$, as wanted.

Now, we are ready to prove Theorem 3.1 .

\section{Proof of Theorem 3.1}

By Lemma 3.7, it is enough to provide $g \in \mathcal{O}\left(\mathbb{R}^{n}\right)$ with $\{g=0\} \subset\{f=0\}$ and a Pfister bundle of rank $p=2^{r+1}$ for $g^{2} f$. We proceed in several steps:

INITIAL PREPARATION. For each $k \geq 1$, there exist, by Theorem 2.12, analytic functions $g_{0 k}, a_{k} \in \mathcal{O}\left(\mathbb{R}^{n}\right)$ such that $\left\{g_{0 k}=0\right\} \subset\left\{a_{k}=0\right\}=\left\{f_{k}=0\right\}$ and $g_{0 k}^{2} f_{k}=a_{k}^{2}+2^{r}$ in $\mathcal{O}\left(\mathbb{R}^{n}\right)$. For each $k \geq 1$ there are, by Lemma 3.3, an analytic function $g_{1 k} \in \mathcal{O}\left(\mathbb{R}^{n}\right)$ with $\left\{g_{1 k}=0\right\} \subset\left\{f_{k}=0\right\}$ and a matrix $M_{k} \in \mathfrak{M}_{p}\left(\mathcal{O}\left(\mathbb{R}^{n}\right)\right)$ such that $M_{k}^{t} M_{k}=h_{k} I_{p}$ where $h_{k}=\left(g_{0 k} g_{1 k}\right)^{2} f_{k}$.

Denote $f=\pi f_{k}$ and $g=\pi\left(g_{0 k} g_{1 k}\right)$ and observe that $\{g=0\} \subset\{f=0\}$ and $h=g^{2} f=\pi h_{k}$ where $h_{k}=\left(g_{0 k} g_{1 k}\right)^{2} f_{k}$. Thus, it is enough to construct a Pfister's bundle $\mathscr{P}(h)=\left(\left(E, \pi, \mathbb{R}^{n}\right), s\right)$ of rank $p$ for $h$.

For each $k \geq 0$ consider the set $W_{k}=\mathbb{R}^{n} \backslash \bigcup_{i>k} X_{i}$. Observe that since the family $\left\{X_{k}\right\}_{k \geq 1}$ is locally finite, each set $W_{k}$ is open in $\mathbb{R}^{n}$ and $\mathbb{R}^{n}=\bigcup_{k \geq 1} W_{k}$.

Step 1: Construction of The Vector Bundle. Note that since $M_{k}^{t} M_{k}=$ $h_{k} I_{p}$, we have that $M_{k}(x) \in \mathrm{GL}\left(\mathbb{R}^{p}\right)$ if and only if $x \in \mathbb{R}^{n} \backslash\left\{h_{k}=0\right\}$. Observe that if $0 \leq i<j$, then $W_{i} \cap W_{j}=W_{i}$ and we define the following transition maps:

$$
\begin{aligned}
& g_{i j}: W_{i} \cap W_{j}=W_{i} \rightarrow \mathrm{GL}\left(\mathbb{R}^{p}\right), x \mapsto \frac{1}{\sqrt{\left(h_{j} \mid W_{i}\right)(x) \cdots\left(h_{i+1} \mid W_{i}\right)(x)}} M_{j}(x) \cdots M_{i+1}(x), \\
& g_{j i}: W_{i} \cap W_{j}=W_{i} \rightarrow \operatorname{GL}\left(\mathbb{R}^{p}\right), x \mapsto \frac{1}{\sqrt{\left(h_{j} \mid W_{i}\right)(x) \cdots\left(h_{i+1}\right) \mid W_{i}(x)}} M_{i+1}^{t}(x) \cdots M_{j}^{t}(x),
\end{aligned}
$$


Obviously, we define $g_{i i}: W_{i} \cap W_{i}=W_{i} \rightarrow \mathrm{GL}\left(\mathbb{R}^{p}\right), x \mapsto I_{p}$. Now, using the fact that $M_{k}^{t} M_{k}=h_{k} I_{p}$ for all $k \geq 1$, we have $g_{j k}(x) \cdot g_{i j}(x)=g_{i k}(x)$ for all $0 \leq i, j, k$ and all $x \in W_{i} \cap W_{j} \cap W_{k}=W_{\min \{i, j, k\}}$. The functions $g_{i j}: W_{i} \cap W_{j} \rightarrow$ $\operatorname{GL}\left(\mathbb{R}^{p}\right)$ are the transition maps of an analytic vector bundle $\left(E, \pi, \mathbb{R}^{n}\right)$ with fiber $\mathbb{R}^{p}$. For each $i \geq 0$ let $\phi_{i}: \pi^{-1}\left(W_{i}\right) \rightarrow W_{i} \times \mathbb{R}^{p}$ be homeomorphisms such that $\left(\phi_{j} \circ \phi_{i}^{-1}\right)(z, v)=\left(z, g_{i j}(z)(v)\right)$ for all $(z, v) \in\left(W_{i} \cap W_{j}\right) \times \mathbb{R}^{p}$ and all $j \geq 0$. Moreover, for each $x \in W_{i}$ and each $j \geq 0$, the matrices $g_{i j}(x) \in \mathrm{GL}\left(\mathbb{R}^{p}\right)$ are in fact ortogonal matrices, that is, $g_{i j}(x) g_{i j}(x)^{t}=I_{p}$.

Step 2: Construction of the Riemaniann metric. Consider the analytic Riemannian metric on $\left(E \oplus E, \pi^{\prime}, \mathbb{R}^{n}\right)$ given by

$$
\langle\cdot, \cdot\rangle \circ \psi_{i}^{-1}\left(x,\left(v_{1}, \ldots, v_{p}\right),\left(w_{1}, \ldots, w_{p}\right)\right)=\sum_{\ell=1}^{p} v_{\ell} w_{\ell},
$$

where $\psi_{i}: \pi^{\prime-1}\left(W_{i}\right) \rightarrow W_{i} \times\left(\mathbb{R}^{p} \oplus \mathbb{R}^{p}\right)$ are homeomorphisms such that

$$
\left(\psi_{j} \circ \psi_{i}^{-1}\right)(z, u, v)=\left(z, g_{i j}(z)(v), g_{i j}(z)(w)\right)
$$

for all $(z, v, w) \in\left(W_{i} \cap W_{j}\right) \times \mathbb{R}^{2 p}$ and all $j \geq 0$. Since each matrix $g_{i j}(x) \in$ $\mathrm{GL}\left(\mathbb{R}^{p}\right)$ is orthogonal, we deduce that $\langle\cdot, \cdot\rangle$ is well-defined.

Step 3: ConstruCtion OF THE ANALYTIC SECTION. Let $u \in \mathbb{R}^{p}$ be any unitary vector and let $s: \mathbb{R}^{n} \rightarrow E$ be the global analytic section satisfying

$$
\left.\phi_{0} \circ s\right|_{W_{0}}: W_{0} \rightarrow W_{0} \times \mathbb{R}^{p} \subset W_{i} \times \mathbb{R}^{p}, x \mapsto\left(x, \sqrt{\left(\left.h\right|_{W_{0}}\right)}(x) u\right) .
$$

Let us check that such section exists. Indeed, for each $i \geq 1$, we have

$\left(\left.\phi_{i} \circ s\right|_{W_{0}}\right)=\left(\phi_{i} \circ \phi_{0}^{-1}\right) \circ\left(\left.\phi_{0} \circ s\right|_{W_{0}}\right): W_{0} \rightarrow W_{0} \times \mathbb{R}^{p}, x \mapsto\left(x, \sqrt{\left(\left.h\right|_{W_{0}}\right)}(x) g_{0 i}(x)(u)\right)$

where

$$
\sqrt{\left.h\right|_{W_{0}}}(x) g_{0 i}(x)(u)=\sqrt{\frac{\left(\left.h\right|_{W_{0}}\right)(x)}{\left(\left.h_{1}\right|_{W_{0}}\right)(x) \cdots\left(h_{i}||_{0}\right)(x)}} M_{i}(x) \cdots M_{1}(x)(u) .
$$

Moreover, in $W_{i}=\mathbb{R}^{n} \backslash \bigcup_{j>i} X_{j}$ the function $\frac{\left.h\right|_{W_{i}}}{\left(\left.h_{1}\right|_{W_{i}}\right) \cdots\left(\left.h_{i}\right|_{W_{i}}\right)}$ is analytic and vanishes nowhere; hence, it admits an analytic square root in $W_{i}$. Since $\mathbb{R}^{n}=\bigcup_{i \geq 0} W_{i}$, we have, by means of equality $(\star)$ above, an analytic section $s: \mathbb{R}^{n} \rightarrow E$ of $\left(E, \pi, \mathbb{R}^{n}\right)$ such that

$$
\phi_{i} \circ s: W_{i} \mapsto W_{i} \times \mathbb{R}^{p}, x \mapsto\left(x, \sqrt{\frac{\left(\left.h\right|_{W_{i}}\right)(x)}{\left(h_{1} \mid W_{i}\right)(x) \cdots\left(\left.h_{i}\right|_{W_{i}}\right)(x)}} M_{i}(x) \cdots M_{1}(x)(u)\right) .
$$

Moreover, the analytic function $\langle s, s\rangle: \mathbb{R}^{n} \rightarrow \mathbb{R}$ is determined by its value on the points $x \in W_{0}$ and so

$$
\left.\langle s, s\rangle\right|_{W_{0}}(x)=\left\langle\sqrt{\left(\left.h\right|_{W_{0}}\right)}(x) u, \sqrt{\left(\left.h\right|_{W_{0}}\right)}(x) u\right\rangle=\left(\left.h\right|_{W_{0}}\right)(x)\langle u, u\rangle=\left(\left.h\right|_{W_{0}}\right)(x) ;
$$

hence, we conclude $\langle s, s\rangle=h$, and we are done. 
Remark 3.8. By means of Remark 3.4(ii), one can adapt the previous proof to show that: If each $f_{k}$ in the statement of Theorem 3.1 is a sum of $2^{r}$ squares in $\mathcal{O}\left(\mathbb{R}^{n}\right)$ with $r=0,1,2,3$, then also $f$ is a sum of $2^{r}$ squares in $\mathcal{O}\left(\mathbb{R}^{n}\right)$. In particular, all the examples in [8, Section 3] are sums of 4 squares of analytic functions on $\mathbb{R}^{n}$.

Finally in this section, we present the proof of Theorem 1.3 as a consequence of Theorem 3.1 with the supplement that the representation as sum of squares of the involved sheaf-product $f$ has controlled denominators.

Proof of Theorem 1.3. First, let $W_{k}$ be an open neighborhood of $\left\{f_{k}=0\right\}$ in $\mathbb{R}^{n}$ and let $\varphi_{k}: M_{k} \rightarrow W_{k}$ be an analytic diffeomorphism such that $M_{k}$ is a Nash manifold and $f_{k} \circ \varphi_{k}=u_{k} g_{k}$, where $g_{k}: M_{k} \rightarrow \mathbb{R}$ is a positive semidefinite Nash function and $u_{k} \in \mathcal{O}\left(M_{k}\right)$ is a positive unit. By Artin-Mazur's Theorem [4, 8.8.4] and [16, 7.4], each Nash function $g_{k}$ is a sum of $2^{n}$ squares with controlled denominators in the quotient field of the ring $\mathcal{N}\left(M_{k}\right)$ of Nash functions on $M_{k}$. Thus, each $f_{k}$ is a sum of $2^{n}$ squares at $\left\{f_{k}=0\right\}$ with controlled denominators; hence, $f$ is, by Theorem 3.1 , a sum of $2^{n+1}$ squares of meromorphic functions with controlled denominators.

\section{Unbounded analytic Pfister formula}

The main purpose of this section is to prove Theorem 1.1(i). We first provide a precise statement of what we will prove.

Theorem 4.1 (Unbounded analytic Pfister formula). Let $\left\{f_{k}\right\}_{k \geq 1}$ be a collection of positive semidefinite analytic functions on $\mathbb{R}^{n}$ such that the family $\left\{X_{k}=\left\{f_{k}=\right.\right.$ $0\}\}_{k \geq 1}$ of their zero sets is locally finite and each $f_{k}$ is a finite sum of squares at $X_{k}$. Then, $\pi f_{k}$ is an infinite sum of squares (with controlled denominators).

We have shown in Lemma 3.7 that if $f \in \mathcal{O}\left(\mathbb{R}^{n}\right)$ is a finite sum of squares, there exist $g \in \mathcal{O}\left(\mathbb{R}^{n}\right) \backslash\{0\}$ and a Pfister bundle $\mathscr{P}\left(g^{2} f\right)$ of finite rank $p$ for $g^{2} f$. In fact, $p$ is an upper bound for the number of squares needed to represent $f$ as a sum of squares. Thus, to approach the unbounded situation presented in Theorem 4.1 , it seems convenient to work with a bundle of infinite rank; an initial candidate for the fiber could be the Hilbert space $\ell^{2}(\mathbb{R})$ of real square-summable sequences. However, since we also need to take care of the uniform convergence of infinite sums of squares, we must work in a complex open Stein neighborhood $\Omega$ of $\mathbb{R}^{n}$ in $\mathbb{C}^{n}$ and we must build up a vector bundle with fiber the complex Hilbert space $\ell^{2}(\mathbb{C})$ of complex square-summable sequences. We begin by fixing and recalling some notation and terminology about Hilbert spaces and vector bundles with fiber a Hilbert space.

\subsection{Hilbert spaces, holomorphic functions and vector bundles}

The Hilbert space $\ell^{2}(\mathbb{C})$ is the vector space

$$
\ell^{2}(\mathbb{C})=\left\{w=\left(w_{k}\right)_{k \geq 1}: \sum_{k \geq 1}\left|w_{k}\right|^{2}<+\infty\right\}
$$


endowed with the usual (hermitian) inner product given by the formula

$$
\langle w, v\rangle_{\mathrm{u}}=\sum_{k \geq 1} w_{k} \overline{v_{k}}
$$

We refer the reader to [19] for the general theory of Hilbert spaces. We denote by $\mathcal{E}=\left\{\mathbf{e}_{m}=\left(\delta_{m k}\right)_{k \geq 1}\right\}_{m \geq 1}$ the standard orthonormal basis of $\ell^{2}(\mathbb{C})$ and by $\mathcal{B}\left(\ell^{2}(\mathbb{C})\right)$ the $\mathbb{C}$-linear space of bounded (linear) operators $\ell^{2}(\mathbb{C}) \rightarrow \ell^{2}(\mathbb{C})$; moreover, the set of those bounded operators which are invertible and whose inverse is also bounded will be denoted by $\mathcal{B}_{*}\left(\ell^{2}(\mathbb{C})\right)$. Given a bounded operator $T \in$ $\mathcal{B}\left(\ell^{2}(\mathbb{C})\right)$, we define its adjoint as the unique bounded operator $T^{*} \in \mathcal{B}\left(\ell^{2}(\mathbb{C})\right)$ such that $\langle v, T(w)\rangle_{\mathrm{u}}=\left\langle T^{*}(v), w\right\rangle_{\mathrm{u}}$ for all $v, w \in \ell^{2}(\mathbb{C})$.

We refer here the reader to [12] and [23, Section 3] for the general theory of holomorphic functions with values in a Banach space and for the general theory of holomorphic Banach vector bundles. Let $\Omega$ be an open subset of $\mathbb{C}^{n}$. From [23, Def. $3.1]$ and the related comments, one deduces almost straightforwardly that a function $F: \Omega \rightarrow \ell^{2}(\mathbb{C})$ is holomorphic if and only if $F$ belongs to the $\mathcal{H}(\Omega)$-module

$$
\left\{F=\left(F_{k}\right)_{k \geq 1}: F_{k} \in \mathcal{H}(\Omega), \sum_{k \geq 1} \sup _{K}\left|F_{k}\right|^{2}<+\infty \forall K \subset \Omega \text { compact }\right\} .
$$

Observe that if $G, H: \Omega \rightarrow \ell^{2}(\mathbb{C})$ are holomorphic, then $\langle G, H \circ \sigma\rangle_{\mathrm{u}}: \Omega \rightarrow$ $\mathbb{C}$ defines an holomorphic function on $\Omega$, which is moreover $\sigma$-invariant, and an infinite sum of squares if $\Omega$ is $\sigma$-invariant and $G=H$.

Proposition and Definition 4.2. Let $M=\left(m_{i j}\right) \in \mathfrak{M}_{p}(\mathbb{C})$ be a matrix and consider the linear operator $T_{M}: \ell^{2}(\mathbb{C}) \rightarrow \ell^{2}(\mathbb{C})$ whose matrix with respect to the standard basis $\mathcal{E}$ of $\ell^{2}(\mathbb{C})$ is the infinite matrix

$$
\left(\begin{array}{c|c|c|c}
M & 0 & 0 & \cdots \\
\hline 0 & M & 0 & \cdots \\
\hline 0 & 0 & M & \cdots \\
\hline \vdots & \vdots & \vdots & \ddots
\end{array}\right) .
$$

Then:

(i) $T_{M}$ is a bounded operator of $\ell^{2}(\mathbb{C})$.

(ii) $T_{M_{1} \cdot M_{2}}=T_{M_{1}} \circ T_{M_{2}}$ for all $M_{1}, M_{2} \in \mathfrak{M}_{p}(\mathbb{C})$.

(iii) $T_{I_{p}}=\mathrm{id}_{\ell^{2}(\mathbb{C})}$, where $I_{p}$ denotes the identity matrix of $\mathfrak{M}_{p}(\mathbb{C})$.

(iv) If $M$ is invertible, then $T_{M}$ is an invertible bounded operator whose inverse is the bounded operator $T_{M^{-1}}$.

(v) $T_{M}^{*}=T_{M^{*}}$, where $M^{*}$ denotes the transpose conjugated of $M$.

We call $T_{M}$ the bounded operator of $\ell^{2}(\mathbb{C})$ associated to $M$. 
Proof of Proposition 4.2. Observe first that the linear operator $T_{M}$ is given by the formula $v=\left(v_{k}\right)_{k \geq 1} \mapsto \sum_{k \geq 1} v_{k} T_{M}\left(\mathbf{e}_{k}\right)$, where

$$
T_{M}\left(\mathbf{e}_{k}\right)=\sum_{i=1}^{p} m_{i j} \mathbf{e}_{p q+i} \quad \text { if } k=p q+j \text { with } q \geq 0 \text { and } 1 \leq j \leq p
$$

Notice that $T_{M}(v)=\left(u_{k}\right)_{k \geq 1}$ is a well defined sequence of complex numbers because $u_{p q+i}=\sum_{j=1}^{p} m_{i j} v_{p q+j}$ for all $q \geq 0,1 \leq i \leq p$. Only the fact that $T_{M}\left(\ell^{2}(\mathbb{C})\right) \subset \ell^{2}(\mathbb{C})$ and the boundness of $T_{M}$ require some comment; all the other statements (ii)-(v) are straightforward. From the inequality

$$
\left\langle T_{M}(v), T_{M}(v)\right\rangle_{\mathrm{u}} \leq p^{3} \max _{1 \leq i, j \leq p}\left\{\left|m_{i j}\right|^{2}\right\}\langle v, v\rangle_{\mathrm{u}} \quad \forall v \in \ell^{2}(\mathbb{C})
$$

follows that $T_{M}(v) \in \ell^{2}(\mathbb{C})$ for all $v \in \ell^{2}(\mathbb{C})$ and that $T_{M}$ is a bounded operator. Thus, we proceed with the proof of $(*)$.

Indeed, denote $m=\max _{1 \leq i, j \leq p}\left\{\left|m_{i j}\right|^{2}\right\}$ and observe first that if $1 \leq r, s \leq p$ and $q \geq 0$,

$$
\begin{aligned}
\left|\left\langle T_{M}\left(\mathrm{e}_{p q+r}\right), T_{M}\left(\mathrm{e}_{p q+s}\right)\right\rangle_{\mathrm{u}}\right| & =\left|\left\langle T_{M}\left(\mathrm{e}_{r}\right), T_{M}\left(\mathrm{e}_{s}\right)\right\rangle_{\mathrm{u}}\right| \\
& =\left|\sum_{i=1}^{p} m_{i r} m_{i s}\right| \\
& \leq \sum_{i=1}^{p}\left|m_{i r} m_{i s}\right| \leq p m .
\end{aligned}
$$

Consider the sequences of positive real numbers $v^{*}=\left(\left|v_{k}\right|\right)_{k \geq 1}$ and $w_{s}=\left(w_{s k}\right)_{k \geq 1}$ for $1 \leq s \leq p$, where $w_{s k}=\left|v_{p j+s}\right|$ if $k=p j+r$ with $1 \leq r \leq p$ and $j \geq 0$. We have

$$
\left\langle v^{*}, v^{*}\right\rangle_{\mathrm{u}}=\sum_{k \geq 1}\left|v_{k}\right|^{2}=\langle v, v\rangle_{\mathrm{u}} \quad \text { and } \quad\left\langle w_{s}, w_{s}\right\rangle_{\mathrm{u}}=p \sum_{j \geq 1}\left|v_{p j+s}\right|^{2} \leq p\langle v, v\rangle_{\mathrm{u}}
$$

hence, in particular $v^{*}, w_{s} \in \ell^{2}(\mathbb{C})$. Moreover,

$$
\left\langle v^{*}, w_{s}\right\rangle_{\mathrm{u}}=\sum_{k \geq 1} v_{k} \overline{w_{s k}}=\sum_{j \geq 0} \sum_{r=1}^{p}\left|v_{p j+r}\right|\left|w_{s, p j+r}\right|=\sum_{j \geq 0} \sum_{r=1}^{p}\left|v_{p j+r}\right|\left|v_{p j+s}\right|
$$

Next, observe that if $\ell_{1}=p q_{1}+r_{1}$ and $\ell_{2}=p q_{2}+r_{2}$ where $1 \leq r_{1}, r_{2} \leq p$ and $q_{1} \neq q_{2}$, then $\left\langle T_{M}\left(\mathrm{e}_{\ell_{1}}\right), T_{M}\left(\mathrm{e}_{\ell_{2}}\right)\right\rangle_{\mathrm{u}}=0$. Thus, for each $q \geq 1$, we have (using 
Schwarz's inequality)

$$
\begin{aligned}
0 & \leq\left|\left\langle\sum_{k=1}^{p q} v_{k} T_{M}\left(\mathrm{e}_{k}\right), \sum_{\ell=1}^{p q} v_{\ell} T_{M}\left(\mathrm{e}_{\ell}\right)\right\rangle_{\mathrm{u}}\right| \\
& =\left|\sum_{k=1}^{p q} \sum_{\ell=1}^{p q} v_{k} \overline{v_{\ell}}\left\langle T_{M}\left(\mathrm{e}_{k}\right), T_{M}\left(\mathrm{e}_{\ell}\right)\right\rangle_{\mathrm{u}}\right| \\
& =\left|\sum_{j=0}^{q} \sum_{r=1}^{p} \sum_{s=1}^{p} v_{p j+r} \overline{v_{p j+s}}\left\langle T_{M}\left(\mathrm{e}_{r}\right), T_{M}\left(\mathrm{e}_{s}\right)\right\rangle_{\mathrm{u}}\right| \\
& \leq \sum_{j=0}^{q} \sum_{r=1}^{p} \sum_{s=1}^{p}\left|v_{p j+r}\right|\left|\overline{v_{p j+s}}\right| p m \\
& =p m \sum_{s=1}^{p}\left(\sum_{j=0}^{q} \sum_{r=1}^{p}\left|v_{p j+r}\right|\left|v_{p j+s}\right|\right) \leq p m \sum_{s=1}^{p}\left\langle v^{*}, w_{s}\right\rangle_{\mathrm{u}} \\
& \leq p m \sum_{s=1}^{p} \sqrt{\left\langle v^{*}, v^{*}\right\rangle_{\mathrm{u}}} \sqrt{\left\langle w_{s}, w_{s}\right\rangle_{\mathrm{u}}} \leq p^{3} m\langle v, v\rangle_{\mathrm{u}} .
\end{aligned}
$$

Since the previous inequality holds for all $q \geq 1$, we have $\left\langle T_{M}(v), T_{M}(v)\right\rangle_{\mathrm{u}} \leq$ $p^{3} m\langle v, v\rangle_{\mathrm{u}}$, and we are done.

Remark 4.3. Let $\Omega \subset \mathbb{C}^{n}$ be a $\sigma$-invariant open set and let $M=\left(m_{i j}\right) \in \mathfrak{M}_{p}(\mathcal{H}(\Omega))$ be a $\sigma$-invariant matrix such that $M(z)^{t} M(z)=I_{p}$ for all $z \in \Omega$. Then, $M^{t} \circ \sigma=$ $\sigma \circ M^{t}$ and so $T_{M(z)}^{*}=T_{M^{t} \circ \sigma(z)}$ and $T_{M \circ \sigma(z)}^{*} \circ T_{M(z)}=T_{M(z)} \circ T_{M \circ \sigma(z)}^{*}=$ id for each $z \in \Omega$.

\subsection{Pfister's multiplicative formulae}

After the introduction of the bounded operator of $\ell_{C}^{2}$ associated to a complex square matrix, we associate to each $\sigma$-invariant sum of squares of $\mathcal{H}(\Omega)$ a $\sigma$-invariant Pfister matrix in $\mathfrak{M}_{2^{r+1}}(\mathcal{H}(\Omega))$ which is almost unitary. This result can be understood as the counterpart of Lemma 3.3 in the setting of this section. More precisely,

Lemma 4.4 (Pfister's complex matrix). Let $\Omega \subset \mathbb{C}^{n}$ be a $\sigma$-invariant open set and let $B \in \mathcal{H}(\Omega)$ be $\sigma$-invariant such that its zeroset does not meet the compact set $K \subset \Omega$. Let $A=2^{r}$ in $\mathcal{H}(\Omega)$ be $\sigma$-invariant. Then, there exists a positive real constant $c>0$ such that for each $d \geq c$, there exist a $\sigma$-invariant $G \in \mathcal{H}(\Omega)$ satisfying $\left\{G^{2}=0\right\} \cap K=\varnothing$ and a $\sigma$-invariant matrix $M \in \mathfrak{M}_{2^{r+1}}(\mathcal{H}(\Omega))$ such that $M^{t} M=G^{2}\left((d B)^{2}+A\right) I_{2^{r+1}}$.

Proof. Indeed, if $r=0, A=A_{0}^{2}$ and taking $c=1+\frac{\max _{K}(|A|)}{\min _{K}\left(|B|^{2}\right)}, G=1$ and

$$
M=\left(\begin{array}{cc}
d B & -A_{0} \\
A_{0} & d B
\end{array}\right) \in \mathfrak{M}_{2}(\mathcal{H}(\Omega))
$$

we are done. 
Assume $r \geq 1$, and let $A_{1}=2^{r-1}$ and $A_{2}=2^{r-1}$ such that $A=A_{1}+A_{2}$. By induction, there are positive real constants $c_{i}>0$ such that for each $d_{i} \geq$ $c_{i}$, there are $\sigma$-invariant $G_{i} \in \mathcal{H}(\Omega)$ satisfying $\left\{G_{i}^{2}\left(\left(d_{i} B\right)^{2}+A_{i}\right)=0\right\} \cap K=$ $\varnothing$ and a $\sigma$-invariant matrix $M_{i} \in \mathfrak{M}_{2^{r}}(\mathcal{H}(\Omega))$ such that $M_{i}^{t} M_{i}=G_{i}^{2}\left(\left(d_{i} B\right)^{2}+\right.$ $\left.A_{i}\right) I_{2^{r}}$. Take $c=\max \left\{c_{0}, \sqrt{2} c_{1}, \sqrt{2} c_{2}\right\}$ where $c_{0}=1+\frac{\max _{K}(|A|)}{\min _{K}\left(|B|^{2}\right)}$, and for each $d \geq c$ consider the $\sigma$-invariant functions $G_{i} \in \mathcal{H}(\Omega)$ and the $\sigma$-invariant matrices $M_{i} \in \mathfrak{M}_{2^{r}}(\mathcal{H}(\Omega))$ corresponding to the constants $d_{i}=\frac{d}{\sqrt{2}} \geq c_{i}$. Next, take $G=G_{1} G_{2}^{2} F_{2}$, where $F_{2}=\left(\left(d_{2} B\right)^{2}+A_{2}\right)$, and

$$
M=\left(\begin{array}{cc}
G_{2}^{2} F_{2} M_{1} & -G_{1} G_{2} F_{2} M_{2} \\
G_{1} G_{2} F_{2} M_{2} & M_{2} M_{1}^{t} M_{2}
\end{array}\right)
$$

and notice that $\left\{G^{2}\left((d B)^{2}+A\right)=0\right\} \cap K=\varnothing$. A straighforward computation shows that these are the $M$ and $G$ we sought.

The following result, of technical nature, assures the existence of $\sigma$-invariant open Stein neighborhoods of $\mathbb{R}^{n}$ in $\mathbb{C}^{n}$ which admit open exhaustions, whose members (not necessarily contractible) have a good behaviour with respect to the square root operator. Namely,

Lemma 4.5. Let $\Omega$ be a $\sigma$-invariant open neighborhood of $\mathbb{R}^{n}$ in $\mathbb{C}^{n}$ and let $\left\{\Delta_{i}\right\}_{i \geq 1}$ be an open covering of $\Omega$ such that $\Delta_{i} \subset \Delta_{i+1}$ for all $i \geq 1$. Then, there exist $a$ $\sigma$-invariant contractible open Stein neighborhood $\Omega^{\prime} \subset \Omega$ of $\mathbb{R}^{n}$ in $\mathbb{C}^{n}$ and an open covering $\left\{\Omega_{i}\right\}_{i \geq 1}$ of $\Omega^{\prime}$ such that

- $\Omega_{i} \subset \Omega_{i+1}$ and $W_{i}=\mathbb{R}^{n} \cap \Delta_{i} \subset \Omega_{i} \subset \Delta_{i}$ for each $i \geq 1$.

- Each $\sigma$-invariant holomorphic unit $u \in \mathcal{H}\left(\Omega_{i}\right)$ admits a $\sigma$-invariant holomorphic square root on $\Omega_{i}$.

Proof. Denote $W_{0}=\varnothing$ and observe that the sets $\left\{W_{i} \backslash W_{i-1}\right\}_{i \geq 1}$ constitute a partition of $\mathbb{R}^{n}$. For each $x=\left(x_{1}, \ldots, x_{n}\right) \in W_{i} \backslash W_{i-1}$ choose a $\sigma$-invariant open polydisc

$$
B_{x}=\left\{z=\left(z_{1}, \ldots, z_{n}\right) \in \mathbb{C}^{n}:\left|z_{i}-x_{i}\right|<\varepsilon_{x}, 1 \leq i \leq n\right\} \subset \Delta_{i}
$$

centered at $x$ and of radius $\varepsilon_{x}>0$. For each $i \geq 1$, define $\Omega_{i}^{\prime}=\bigcup_{x \in W_{i}} B_{x}$ which is a $\sigma$-invariant open neighborhood of $W_{i}$ in $\Delta_{i}$. Of course, since $W_{i} \subset W_{i+1}$ for $i \geq 1$, also $\Omega_{i}^{\prime} \subset \Omega_{i+1}^{\prime}$. Moreover, $\mathbb{R}^{n}=\bigcup_{i \geq 1} W_{i} \subset \bigcup_{i \geq 1} \Omega_{i}^{\prime} \subset \bigcup_{i \geq 1} \Delta_{i}=\Omega$.

Let $u \in \mathcal{H}\left(\Omega_{i}^{\prime}\right)$ be a $\sigma$-invariant holomorphic unit. For each $x \in W_{i}$ the restriction $\left.u\right|_{B_{x}}$ admits a $\sigma$-invariant holomorphic square root $v_{x} \in \mathcal{H}\left(B_{x}\right)$ whose restriction to $B_{x} \cap \mathbb{R}^{n}$ is strictly positive. Now, by the Identity Principle, the functions $v_{x}$ glue properly to provide a $\sigma$-invariant (no-where vanishing) holomorphic function on $\Omega_{i}^{\prime}$

$$
v: \Omega_{i}^{\prime} \rightarrow \mathbb{C}, z \mapsto v_{x}(z) \text { if } z \in B_{x},
$$

satisfying $v^{2}=u$. Thus, $u$ admits a $\sigma$-invariant holomorphic square root in $\Omega_{i}^{\prime}$. 
Finally, we choose a $\sigma$-invariant contractible open Stein neighborhood $\Omega^{\prime} \subset$ $\bigcup_{i \geq 1} \Omega_{i}^{\prime}$ of $\mathbb{R}^{n}$ in $\mathbb{C}^{n}$. A straightforward computation shows that the $\sigma$-invariant open sets $\Omega_{i}=\Omega_{i}^{\prime} \cap \Omega^{\prime}$ are those we sought.

After all the previous preparation we are ready to prove Theorem 4.1.

\section{Proof of Theorem 4.1}

The proof is rather similar to that of Theorem 3.1; but since this proof is quite more involved and the details are quite more technical, we include it in full detail. The proof runs in several steps:

INITIAL PREPARATION. First, we may assume that $p_{k}=2^{r_{k}}$ and we write $q_{k}=$ $2^{n+1} p_{k}$. By Lemma 2.14, there exist a $\sigma$-invariant open neighborhood $\Omega$ of $\mathbb{R}^{n}$ in $\mathbb{C}^{n}$ and $\sigma$-invariant homolomorphic functions $G_{k}, \widehat{F}_{k}, A_{k j}: \Omega \rightarrow \mathbb{C}$ for $k \geq 1$ and $1 \leq j \leq q_{k}$ such that:

(i) $\left.\widehat{F}_{k}\right|_{\mathbb{R}^{n}}=u_{k} f_{k}$ for a positive unit $u_{k} \in \mathcal{O}\left(\mathbb{R}^{n}\right)$.

(ii) The family $\left\{\left\{G_{k}^{2} \widehat{F}_{k}=0\right\}\right\}_{k \geq 1}$ is locally finite in $\Omega$ and $\left\{G_{k}^{2} \widehat{F}_{k}=0\right\} \cap \mathbb{R}^{n}=X_{k}$.

(iii) For each family of values $\left\{d_{k}\right\}_{k \geq 1}$ satisfying $d_{k} \geq c_{k}$, the finite sums $A_{k}=$ $\left(d_{k} G_{k}^{2} \widehat{F}_{k}\right)^{2}+\sum_{j=1}^{q_{k}} A_{k j}^{2}$ satisfy

- $\left\{\left.A_{k}\right|_{\mathbb{R}^{n}}=0\right\}=X_{k}$ and the family $\left\{\left\{A_{k}=0\right\}\right\}_{k \geq 1}$ is locally finite in $\Omega$; and

- $A_{k}=G_{k}^{2} \widehat{F}_{k} Q_{k}$ for some $\sigma$-invariant $Q_{k} \in \mathcal{H}(\Omega)$, which does not vanish on a neighborhood $V_{k}$ of $\left\{G_{k}^{2} \widehat{F}_{k}=0\right\} \cup \mathbb{R}^{n}$.

Since the family $\left\{\left\{G_{k}^{2} \widehat{F}_{k}=0\right\}\right\}_{k \geq 1}$ is locally finite, there exists, by Lemma 2.7, an exhaustion $\left\{K_{k}\right\}_{k \geq 1}$ of $\Omega$ by compact sets such that $\left\{G_{k}^{2} \widehat{F}_{k}=0\right\} \cap K_{k}=\varnothing$ for all $k \geq 1$. By Lemma 4.4, applied to $B_{k}=G_{k}^{2} \widehat{F}_{k}$, the sum of squares $\sum_{j=1}^{q_{k}} A_{k j}^{2}$ and the compact set $K_{k}$, there exist a real number $d_{k} \geq c_{k}$, a $\sigma$-invariant $G_{k}^{\prime} \in \mathcal{H}(\Omega)$ satisfying $\left\{G_{k}^{\prime 2}=0\right\} \cap K_{k}=\varnothing$ and a $\sigma$-invariant matrix $M_{k} \in \mathfrak{M}_{2 q_{k}}(\mathcal{H}(\Omega))$ such that $M_{k}^{t} M_{k}=G_{k}^{\prime 2} A_{k} I_{2 q_{k}}$, where $A_{k}=\left(d_{k} B_{k}\right)^{2}+\sum_{j=1}^{q_{k}} A_{k j}^{2}$.

To simplify notation, we denote again by $G_{k}$ the function $G_{k} G_{k}^{\prime}$ and by $A_{k}$ the function $G_{k}^{\prime 2} A_{k}$. Observe that, by Lemma 2.7, the family $\left\{\left\{G_{k}=0\right\}\right\}_{k \geq 1}$ is locally finite. Let $\widehat{F}=\pi \widehat{F}_{k}, G=\pi G_{k}$ and $A=\pi A_{k}$ and denote $f=\left.\widehat{F}\right|_{\mathbb{R}^{n}}, g=\left.G\right|_{\mathbb{R}^{n}}$ and $a=\left.A\right|_{\mathbb{R}^{n}}$. Observe that $f=\pi f_{k}$ and in fact, by (iii) above, $g^{2} f=u a$ for a positive unit $u \in \mathcal{O}\left(\mathbb{R}^{n}\right)$. Thus, we are reduced to prove that $A$ is an infinite sum of squares of $\sigma$-invariant holomorphic functions on $\Omega$ and this is done in the subsequent steps.

Step 2: Construction OF A SUITABle VeCTOR BUNDLE With Fiber $\ell^{2}(\mathbb{C})$. For each $i \geq 0$, denote by $\Delta_{i}$ the open subset $\Omega \backslash \bigcup_{k>i}\left\{A_{k}=0\right\}$ of $\mathbb{C}^{n}$. By Lemma 4.5 , there exist a $\sigma$-invariant contractible open Stein neighborhood $\Omega^{\prime} \subset \Omega$ of $\mathbb{R}^{n}$ in 
$\mathbb{C}^{n}$ and an open covering $\left\{\Omega_{i}\right\}_{i \geq 1}$ of $\Omega^{\prime}$ such that

- $\Omega_{i} \subset \Omega_{i+1}$ and $W_{i}=\mathbb{R}^{n} \cap \Delta_{i} \subset \Omega_{i} \subset \Delta_{i}$ for each $i \geq 0$.

- Each $\sigma$-invariant holomorphic unit $u \in \mathcal{H}\left(\Omega_{i}\right)$ admits a $\sigma$-invariant holomorphic square root on $\Omega_{i}$.

In particular, the restrictions to $\Omega_{i}$ of the quotients (which vanish nowhere in $\Omega_{i}$ )

$$
H_{i}=\frac{A}{A_{1} \cdots A_{i}} \quad \text { and } \quad B_{i j}=\frac{1}{A_{i+1} \cdots A_{j}}
$$

admit $\sigma$-invariant holomorphic square roots in $\Omega_{i}$ for $0 \leq i<j$. To simplify notations, we denote $\Omega^{\prime}$ again by $\Omega$.

For each $k \geq 0$ consider the product $\Omega_{k} \times \ell^{2}(\mathbb{C})$. Since the $\sigma$-invariant matrix $M_{k} \in \mathfrak{M}_{2 q_{k}}(\mathcal{H}(\Omega))$ constructed in the previous step satisfies $M_{k}^{t} M_{k}=A_{k} I_{2 q_{k}}$, we deduce that $T_{M_{k}(z)}: \ell^{2}(\mathbb{C}) \rightarrow \ell_{C}^{2}$ is an isomorphism for each $z \in \Omega \backslash\left\{A_{k}=0\right\}$.

Observe that if $0 \leq i<j$, then $\Omega_{i} \cap \Omega_{j}=\Omega_{i}$ and we define the following transition functions:

$$
\begin{aligned}
& \gamma_{i j}: \Omega_{i} \cap \Omega_{j}=\Omega_{i} \rightarrow \mathcal{B}_{*}\left(\ell^{2}(\mathbb{C})\right), z \mapsto \sqrt{B_{i j} \mid \Omega_{i}(z)} T_{M_{j}(z)} \circ \cdots \circ T_{M_{i+1}(z)}, \\
& \gamma_{j i}: \Omega_{i} \cap \Omega_{j}=\Omega_{i} \rightarrow \mathcal{B}_{*}\left(\ell^{2}(\mathbb{C})\right), z \mapsto \sqrt{\left.B_{i j}\right|_{\Omega_{i}}(z)} T_{M_{i+1} \circ \sigma(z)}^{*} \circ \cdots \circ T_{M_{j} \circ \sigma(z)}^{*} .
\end{aligned}
$$

Obviously, we define $\gamma_{i i}: \Omega_{i} \cap \Omega_{i}=\Omega_{i} \rightarrow \mathcal{B}_{*}\left(\ell^{2}(\mathbb{C})\right), z \mapsto$ id. Now, using the fact that

$$
T_{M_{k} \circ \sigma(z)}^{*} \circ T_{M_{k}(z)}=T_{M_{k}(z)} \circ T_{M_{k} \circ \sigma(z)}^{*}=A_{k}(z) \text { id }
$$

for all $k \geq 1$ and all $z \in \Omega$, one checks that $\gamma_{j k}(z) \cdot \gamma_{i j}(z)=\gamma_{i k}(z)$ for all $0 \leq$ $i, j, k$ and all $z \in \Omega_{i} \cap \Omega_{j} \cap \Omega_{k}=\Omega_{\min \{i, j, k\}}$. Thus, the holomorphic functions $\gamma_{i j}: \Omega_{i} \cap \Omega_{j} \rightarrow \mathcal{B}_{*}\left(\ell^{2}(\mathbb{C})\right)$ are the transition functions of a holomorphic Hilbert vector bundle $\mathscr{E}=(E, \pi, \Omega)$ with fiber $\ell^{2}(\mathbb{C})$ (see [23, Section 1, Section 3]). For each $i \geq 0$ denote by $\psi_{i}: \pi^{-1}\left(\Omega_{i}\right) \rightarrow \Omega_{i} \times \ell^{2}(\mathbb{C})$ homeomorphisms such that $\psi_{j} \circ \psi_{i}^{-1}(z, v)=\left(z, \gamma_{i j}(z)(v)\right)$ for all $(z, v) \in \Omega_{\min \{i, j\}} \times \ell^{2}(\mathbb{C})$ and all $i, j \geq 0$. Moreover, observe that for each $z \in \Omega_{i}$ and each $j \geq 0$, the bounded operators $\gamma_{i j}(z) \in \mathcal{B}_{*}\left(\ell^{2}(\mathbb{C})\right)$ satisfy the equality

$$
\gamma_{i j}(z) \cdot \gamma_{i j}^{*}(\sigma(z))=\gamma_{i j}^{*}(\sigma(z)) \cdot \gamma_{i j}(z)=\mathrm{id} .
$$

Step 3: Construction of a suitable Hermitian structure. Next, consider the Whitney sum $\mathscr{E} \oplus \mathscr{E}^{\sigma}=\left(E \oplus E^{\sigma}, \pi^{\prime}, \Omega\right)$ of $\mathscr{E}=(E, \pi, \Omega)$ with $\mathscr{E}^{\sigma}=\left(E^{\sigma}, \pi, \Omega\right)$, where this last vector bundle corresponds to the antiholomorphic structure of $\mathscr{E}$. Recall that given $0 \leq i, j$ the transition function of $\mathscr{E} \oplus \mathscr{E}^{\sigma}$ corresponding to the intersection $\Omega_{i} \cap \Omega_{j}$ is

$$
\Gamma_{i j}: \Omega_{i} \cap \Omega_{j} \rightarrow \mathcal{B}_{*}\left(\ell^{2}(\mathbb{C}) \oplus \ell^{2}(\mathbb{C})\right), z \mapsto \gamma_{i j}(z) \oplus \gamma_{i j}(\sigma(z)) .
$$


For each $i \geq 0$ denote by $\Psi_{i}: \pi^{\prime-1}\left(\Omega_{i}\right) \rightarrow \Omega_{i} \times\left(\ell^{2}(\mathbb{C}) \oplus \ell^{2}(\mathbb{C})\right)$ homeomorphisms such that $\Psi_{j} \circ \Psi_{i}^{-1}(z, v)=\left(z, \Gamma_{i j}(z)(v, w)\right)$ for all $(z, v, w) \in \Omega_{\min \{i, j\}} \times\left(\ell^{2}(\mathbb{C}) \oplus\right.$ $\ell^{2}(\mathbb{C})$ ) and all $i, j \geq 1$. We define the Hermitian metric as follows. For each $i \geq 0$ and each $z \in \Omega_{i}$, consider

$$
\langle\cdot, \cdot\rangle \circ \Psi_{i}^{-1}: \Omega_{i} \times\left(\ell^{2}(\mathbb{C}) \times \ell^{2}(\mathbb{C})\right) \rightarrow \mathbb{C},(z, v, w) \mapsto\langle v, w\rangle_{\mathrm{u}} .
$$

Let us see that $\langle\cdot, \cdot\rangle$ is well defined. To that end, fix $i, j \geq 1$ and $(z, v, w) \in$ $\Omega_{\min \{i, j\}} \times\left(\ell^{2}(\mathbb{C}) \oplus \ell^{2}(\mathbb{C})\right)$ and let us check the following equality

$$
\langle\cdot, \cdot\rangle \circ \Psi_{j}^{-1}(z, v, w)=\langle\cdot, \cdot\rangle \circ \Psi_{i}^{-1}(z, v, w) .
$$

Indeed, using $(*)$ above we deduce

$$
\begin{aligned}
\langle\cdot, \cdot\rangle \circ \Psi_{j}^{-1}(z, v, w) & =\langle\cdot, \cdot\rangle \circ\left(\Psi_{i}^{-1} \circ \Psi_{i}\right) \circ \Psi_{j}^{-1}(z, v, w) \\
& =\langle\cdot, \cdot\rangle \circ \Psi_{i}^{-1} \circ \Gamma_{i j}(z, v, w) \\
& =\langle\cdot, \cdot\rangle \circ \Psi_{i}^{-1}\left(z, \gamma_{i j}(z)(v),\left(\gamma_{i j} \circ \sigma\right)(z)(w)\right) \\
& =\left\langle\gamma_{i j}(z)(v),\left(\gamma_{i j} \circ \sigma\right)(z)(w)\right\rangle_{\mathrm{u}} \\
& =\left\langle\gamma_{i j}^{*}(\sigma(z)) \cdot \gamma_{i j}(z)(v), w\right\rangle_{\mathrm{u}} \\
& =\langle v, w\rangle_{\mathrm{u}}=\langle\cdot, \cdot\rangle \circ \Psi_{i}^{-1}(z, v, w) .
\end{aligned}
$$

Moreover, given holomorphic sections $\alpha, \beta: \Omega \rightarrow \mathscr{E}$, we define in the obvious way the section $\alpha \oplus(\beta \circ \sigma): \Omega \rightarrow \mathscr{E} \oplus \mathscr{E}^{\sigma}$, to which we can apply $\langle\cdot, \cdot\rangle: \mathscr{E} \oplus \mathscr{E} \sigma \rightarrow \mathbb{C}$, and we get the holomorphic function $\langle\alpha, \beta \circ \sigma\rangle: \Omega \rightarrow \mathbb{C}$.

Step 4: Construction of Suitable holomorphic sections. Denote by $\mathcal{E}=\left\{\mathbf{e}_{\ell}\right\}_{\ell \geq 1}$ the standard basis of $\ell^{2}(\mathbb{C})$. Let us construct holomorphic sections $s_{\ell}: \Omega \rightarrow \mathscr{E}$ such that

$$
\left.\psi_{0} \circ s_{\ell}\right|_{\Omega_{0}}: \Omega_{0} \rightarrow \Omega_{0} \times \ell^{2}(\mathbb{C}) \subset \Omega_{i} \times \ell^{2}(\mathbb{C}), z \mapsto\left(z, \sqrt{\left.A\right|_{\Omega_{0}}}(z) \mathbf{e}_{\ell}\right)
$$

Indeed, since $A$ vanishes nowhere in $\Omega_{0}$, the square root $\sqrt{\left.A\right|_{\Omega_{0}}}$ is well-defined and holomorphic in $\Omega_{0}$. For each $i \geq 1$, we have

$$
\begin{aligned}
& \left(\left.\psi_{i} \circ s_{\ell}\right|_{\Omega_{0}}\right) \\
& =\left(\psi_{i} \circ \psi_{0}^{-1}\right) \circ\left(\left.\psi_{0} \circ s_{\ell}\right|_{\Omega_{0}}\right): \Omega_{0} \rightarrow \Omega_{0} \times \ell^{2}(\mathbb{C}), z \mapsto\left(z, \sqrt{\left.A\right|_{\Omega_{0}}}(z) \gamma_{0 i}(z)\left(\mathbf{e}_{\ell}\right)\right)
\end{aligned}
$$

where

$$
\sqrt{\left.A\right|_{\Omega_{0}}}(z) \gamma_{0 i}(z)\left(\mathbf{e}_{\ell}\right)=\sqrt{\left.H_{i}\right|_{\Omega_{0}}(z)}\left(T_{M_{i}(z)} \circ \cdots \circ T_{M_{1}(z)}\right)\left(\mathbf{e}_{\ell}\right) .
$$

Moreover, the square roots $\sqrt{\left.H_{i}\right|_{\Omega_{i}}}: \Omega_{i} \rightarrow \mathbb{C}$ are well-defined and holomorphic on $\Omega_{i}$. Since $\Omega=\bigcup_{i \geq 0} \Omega_{i}$, we define $s_{\ell}: \Omega \rightarrow \mathscr{E}$ such that

$$
\psi_{i} \circ s_{\ell}: \Omega_{i} \rightarrow \Omega_{i} \times \ell^{2}(\mathbb{C}), z \mapsto\left(z, \sqrt{\left.H_{i}\right|_{\Omega_{i}}(z)}\left(T_{M_{i}(z)} \circ \cdots \circ T_{M_{1}(z)}\right)\left(\mathbf{e}_{\ell}\right)\right)
$$


which is a holomorphic section of $\mathscr{E}$. Moreover, consider the holomorphic functions $\left\langle s_{k}, s_{\ell} \circ \sigma\right\rangle: \Omega \rightarrow \mathbb{C}$. Notice that, by the Identity Principle, the holomorphic functions $\left\langle s_{k}, s_{\ell} \circ \sigma\right\rangle: \Omega \rightarrow \mathbb{C}$ are determined by their value on the points $z \in \Omega_{0}$. Thus, since

$$
\begin{aligned}
\left.\left\langle s_{k}, s_{\ell} \circ \sigma\right\rangle\right|_{\Omega_{0}}(z) & =\left\langle\left(\psi_{0} \circ s_{k}\right)(z),\left(\psi_{0} \circ s_{\ell} \circ \sigma\right)(z)\right\rangle_{\mathrm{u}} \\
& =\left\langle\sqrt{\left.A\right|_{\Omega_{0}}}(z) \mathbf{e}_{k}, \sqrt{\left.A\right|_{\Omega_{0}}} \circ \sigma(z) \mathbf{e}_{\ell}\right\rangle_{\mathrm{u}} \\
& =\left(\sqrt{\left.A\right|_{\Omega_{0}}}(z)\right)^{2}\left\langle\mathbf{e}_{k}, \mathbf{e}_{\ell}\right\rangle_{\mathrm{u}} \\
& =\left.A\right|_{\Omega_{0}}(z) \delta_{k \ell},
\end{aligned}
$$

we deduce that $\left\langle s_{k}, s_{\ell} \circ \sigma\right\rangle=A \delta_{k \ell}$ for $k, \ell \geq 1$.

Step 5: REPRESENTATION OF A AS A SUM OF SQUARES. First, since the fiber bundle is trivial on $\Omega$, there exists, by [23,3.13], a nowhere-vanishing holomorphic section $\tau: \Omega \rightarrow E$; hence, the restriction to $\mathbb{R}^{n}$ of the $\sigma$-invariant holomorphic function $u=\langle\tau, \tau \circ \sigma\rangle: \Omega \rightarrow \mathbb{C}$ is strictly positive. Thus, shrinking $\Omega$ if necessary (but keeping all its properties) there exists a no-where vanishing $\sigma$-invariant holomorphic function $v: \Omega \rightarrow \mathbb{C}$ such that $u=v^{2}$.

Next, let us check that on $\Omega^{*}=\Omega \backslash\{A=0\}$ the following equality holds

$$
\left.\left.A\right|_{\Omega^{*}} \tau\right|_{\Omega^{*}}=\left.\left.\sum_{\ell \geq 1}\left\langle\tau, s_{\ell} \circ \sigma\right\rangle\right|_{\Omega^{*}} S_{\ell}\right|_{\Omega^{*}}
$$

Indeed, since $\Omega^{*}=\bigcup_{i \geq 0} \Omega_{i}^{*}$, where $\Omega_{i}^{*}=\Omega^{*} \cap \Omega_{i}$, it is enough to check that

$$
\left.\left.A\right|_{\Omega_{i}^{*}} \tau\right|_{\Omega_{i}^{*}}=\left.\left.\sum_{\ell \geq 1}\left\langle\tau, s_{\ell} \circ \sigma\right\rangle\right|_{\Omega_{i}^{*}} s_{\ell}\right|_{\Omega_{i}^{*}}
$$

for all $i \geq 0$. Let $\eta_{i}: \Omega_{i}^{*} \rightarrow \ell^{2}(\mathbb{C})$ be a nowhere vanishing holomorphic function such that $\left.\psi_{i} \circ \tau\right|_{\Omega_{i}^{*}}(z)=\left(z, \eta_{i}(z)\right)$ for each $z \in \Omega_{i}^{*}$. Consider the holomorphic function

$$
\beta_{i}: \Omega_{i}^{*} \rightarrow \ell^{2}(\mathbb{C}), z \mapsto \frac{B_{0 i}(z)}{\sqrt{H_{i} \mid \Omega_{i}(z)}}\left(T_{M_{1}(z)}^{*} \circ \cdots \circ T_{M_{i}(z)}^{*}\right)\left(\eta_{i}(z)\right)
$$

and write $\beta_{i}=\sum_{\ell \geq 1} \beta_{i \ell} \mathbf{e}_{\ell}$, where $\beta_{i \ell} \in \mathcal{H}\left(\Omega_{i}^{*}\right)$ and $\sum_{\ell \geq 1} \sup _{K}\left|\beta_{i \ell}\right|^{2}<+\infty$ for all $K \subset \Omega_{i}^{*}$ compact. Then,

$$
\begin{aligned}
\left.\psi_{i} \circ \tau\right|_{\Omega_{i}^{*}}(z) & =\left(z, \eta_{i}(z)\right)=\left(z, \sqrt{\left.H_{i}\right|_{\Omega_{i}}(z)}\left(T_{M_{i}(z)} \circ \cdots \circ T_{M_{1}(z)}\right)\left(\beta_{i}(z)\right)\right) \\
& =\left(z, \sum_{k \geq 1} \beta_{i k}(z)\left(\sqrt{\left.H_{i}\right|_{\Omega_{i}}(z)}\left(T_{M_{i}(z)} \circ \cdots \circ T_{M_{1}(z)}\right)\left(\mathrm{e}_{k}\right)\right)\right) \\
& =\sum_{\ell \geq 1} \beta_{i \ell}(z)\left(\left.\psi_{i} \circ s_{\ell}\right|_{\Omega_{i}^{*}}\right)(z) ;
\end{aligned}
$$


hence, $\left.\tau\right|_{\Omega_{i}^{*}}=\left.\sum_{\ell \geq 1} \beta_{i \ell}(z) s_{\ell}\right|_{\Omega_{i}^{*}}$. Now, using the fact that $\left\langle s_{k}, s_{\ell} \circ \sigma\right\rangle=A \delta_{k \ell}$ for $k, \ell \geq 1$, we deduce that

$$
\left\langle\left.\tau\right|_{\Omega_{i}^{*}},\left.s_{k} \circ \sigma\right|_{\Omega_{i}^{*}}\right\rangle=\left\langle\left.\sum_{\ell \geq 1} \beta_{i \ell}(z) s_{\ell}\right|_{\Omega_{i}^{*}},\left.s_{k} \circ \sigma\right|_{\Omega_{i}^{*}}\right\rangle=\beta_{i k}\left\langle\left. s_{k}\right|_{\Omega_{i}^{*}},\left.s_{k} \circ \sigma\right|_{\Omega_{i}^{*}}\right\rangle=\left.\beta_{i k} A\right|_{\Omega_{i}^{*}},
$$

from which follows equality $(\star)$ and so $(\bullet)$. hence,

Extending formula $(\bullet)$ by zero, we deduce that $A \tau=\sum_{\ell \geq 1}\left\langle\tau, s_{\ell} \circ \sigma\right\rangle s_{\ell}$;

$$
\begin{aligned}
A v^{2} & =A\langle\tau, \tau \circ \sigma\rangle=\langle A \tau, \tau \circ \sigma\rangle \\
& =\sum_{\ell \geq 1}\left\langle\tau, s_{\ell} \circ \sigma\right\rangle\left\langle s_{\ell}, \tau \circ \sigma\right\rangle \\
& =\sum_{\ell \geq 1}\left\langle\tau, s_{\ell} \circ \sigma\right\rangle\left(\overline{\left\langle\tau, s_{\ell} \circ \sigma\right\rangle} \circ \sigma\right)
\end{aligned}
$$

Therefore, since each summand $\left\langle\tau, s_{\ell} \circ \sigma\right\rangle\left(\overline{\left\langle\tau, s_{\ell} \circ \sigma\right\rangle} \circ \sigma\right)$ is a sum of two squares of $\sigma$-invariant holomorphic functions on $\Omega$, we conclude that $A$ is an infinite sum of squares of $\sigma$-invariant holomorphic functions on $\Omega$, as wanted.

\section{Infinite sums of squares}

In this section we prove the following result, from which Theorem 1.1(iii) follows straightforwardly, and which generalizes statement $[8,1.4]$ for dimension $n$.

Theorem 5.1. Let $\left\{f_{k}\right\}_{k \geq 1}$ be a collection of positive semidefinite analytic functions on $\mathbb{R}^{n}$ such that the family $X_{k}=\left\{f_{k}=0\right\}$ of their zero sets is locally finite in $\mathbb{R}^{n}$ and each $f_{k}$ is an infinite sum of squares at $X_{k}$. Let $\left\{Y_{i}\right\}_{i \geq 1}$ be the collection of the global irreducible components (in the sense of [22]) of the global analytic set $X=\bigcup_{k \geq 1} X_{k}$ and for each $i \geq 1$ define

$$
\mathfrak{F}_{i}=\left\{k \geq 1: Y_{i} \cap X_{k} \neq \varnothing\right\} \quad \text { and } \quad I=\left\{i \geq 1: \# \mathfrak{F}_{i}=+\infty\right\} .
$$

Then, there exist a positive semidefinite analytic function $q$ on $\mathbb{R}^{n}$ such that:

(i) $\{q=0\} \subset \bigcup_{j, \ell \in I, j \neq \ell}\left(Y_{j} \cap Y_{\ell}\right)$.

(ii) $q \pi f_{k}$ is an infinite sum of squares with controlled denominators.

\section{Remark 5.2.}

(i) If each irreducible component $Y_{i}$ is compact, then $I=\varnothing$ and $q$ is just a positive unit of $\mathcal{O}\left(\mathbb{R}^{n}\right)$. Hence, $f$ is an infinite sum of squares of analytic functions on $\mathbb{R}^{n}$; see Example 6.2, for an example of a special factor whose zeroset has countably many global irreducible components and all of them are compact. 
(ii) If for each $j, \ell \in I$ with $j \neq \ell$ the intersection $Y_{j} \cap Y_{\ell}$ is a discrete set, then $f$ is an infinite sum of squares on $\mathbb{R}^{n}$. This follows from the fact that $\{q=0\}$ is contained in a discrete set and so, by [5, Theorem 1] or Theorem 1.4, $q$ is a finite sum of squares.

(iii) If $n=3$, then $\operatorname{dim} Y_{i}=1$ for all $i \geq 1$; hence, each intersection $Y_{j} \cap Y_{\ell}$ with $j \neq \ell$ is a discrete set and so, by (ii), $f$ is an infinite sum of squares (see also [8, Section 1, pag. 72]).

(iv) Let $f: \mathbb{R}^{n} \rightarrow \mathbb{R}$ be a positive semidefinite analytic function and suppose that there exist non-identically zero positive semidefinite analytic functions $q_{1}, q_{2} \in \mathcal{O}\left(\mathbb{R}^{n}\right)$ such that $h_{1}=f q_{1}$ and $h_{2}=f q_{2}$ are infinite sum of squares Then, $q_{1}$ is an infinite sum of squares if and only if so is $q_{2}$. This, follows straightforwardly from the fact that $q_{1} q_{2} f^{2}=h_{1} h_{2}$ is an infinite sum of squares.

\section{Proof of Theorem 5.1}

The proof runs in several steps:

REDUCTION: For our purposes it is enough to construct analytic functions $g, h, q$ (which are the restrictions to $\mathbb{R}^{n}$ of $\sigma$-invariant holomorphic functions on a suitable $\sigma$-invariant open neighborhood $\Omega$ of $\mathbb{R}^{n}$ in $\mathbb{C}^{n}$ ) satisfying the following conditions:

- $\{g=0\} \subset X$.

- $h$ is an infinite sum of squares of analytic functions on $\mathbb{R}^{n}$ such that $\{h=0\} \subset$ $X$.

- $q$ is positive semidefinite and $\{q=0\} \subset \bigcup_{j, \ell \in I, j \neq \ell}\left(Y_{j} \cap Y_{\ell}\right)$.

- $g^{2} \pi f_{k}=q h$.

STEP 1: INITIAL PREPARATION OF REAL AND COMPLEX DATA. First, by Lemma 2.14 , there are a $\sigma$-invariant open neighborhood $\Omega$ of $\mathbb{R}^{n}$ in $\mathbb{C}^{n}$, positive real constants $c_{k}$ for $k \geq 1$ and $\sigma$-invariant homolomorphic functions $G_{k}, \widehat{F}_{k}, A_{k j} \in \mathcal{H}(\Omega)$ for $k, j \geq 1$ such that:

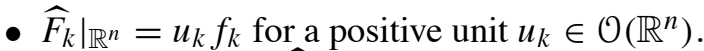

- The family $\left\{\left\{G_{k}^{2} \widehat{F}_{k}=0\right\}\right\}_{k \geq 1}$ is locally finite in $\Omega$ and $\left\{G_{k}^{2} \widehat{F}_{k}=0\right\} \cap \mathbb{R}^{n}=X_{k}$.

- The sum $A_{k}=\left(c_{k} G_{k}^{2} \widehat{F}_{k}\right)^{2}+\sum_{j=1}^{p_{k}} A_{k j}^{2}$ converges in $\Omega$ in the sense of Definition 2.10(ii);

- $\left\{A_{k} \mid \mathbb{R}^{n}=0\right\}=X_{k}$ and the family $\left\{\left\{A_{k}=0\right\}\right\}_{k \geq 1}$ is locally finite in $\Omega$; and

- $A_{k}=G_{k}^{2} \widehat{F}_{k} Q_{k}$ for some $\sigma$-invariant $Q_{k} \in \mathcal{H}(\Omega)$, which does not vanish on a neighborhood $V_{k}$ of $\left\{G_{k}^{2} \widehat{F}_{k}=0\right\} \cup \mathbb{R}^{n}$.

Denote $\widehat{F}=\pi \widehat{F}_{k}, G_{0}=\pi G_{k}, \widehat{f}=\left.\widehat{F}\right|_{\mathbb{R}^{n}}$ and $g_{0}=\left.G_{0}\right|_{\mathbb{R}^{n}}$. Since each $f_{k}$ is positive semidefinite, so is each $\widehat{F}_{k} \mid \mathbb{R}^{n}$ and we may assume that $\widehat{f}$ is positive semidefinite. In fact, since $\widehat{F}_{k} \mid \mathbb{R}^{n}=u_{k} f_{k}$ for each $k \geq 1$, there exists a positive unit $u \in \mathcal{O}\left(\mathbb{R}^{n}\right)$ such that $\widehat{f}=u f$. Observe also that $\left\{g_{0}=0\right\} \subset \bigcup_{k \geq 1} X_{k}=X$. 
Next, for each $i \geq 1$, we define $\mathfrak{G}_{i}=\left\{k \geq 1: Y_{i} \subset X_{k}\right\}$ and notice that $\mathfrak{G}_{i}$ is a finite set for each $i \geq 1$. Now, for each $i \geq 1$, consider

$$
\mathfrak{H}_{i}= \begin{cases}\mathfrak{F}_{i} & \text { if } \mathfrak{F}_{i} \text { is finite, } \\ \mathfrak{G}_{i} & \text { otherwise }\end{cases}
$$

and define $P_{i}=\prod_{k \in \mathfrak{H}_{i}} G_{k}^{2} \widehat{F}_{k}$ and $B_{i}=\prod_{k \in \mathfrak{H}_{i}} A_{k}$, which is a convergent sum of squares $\sum_{\ell \geq 1} B_{i \ell}^{2}$ on $\Omega$ in the sense of Definition 2.10(ii). Of course, each function $P_{i}$ divides $G_{0}^{2} \widehat{F}$ in $\mathcal{H}(\Omega)$ for all $i \geq 1$. Moreover, $B_{i}=P_{i} \prod_{k \in \mathfrak{H}_{i}} Q_{k}=P_{i} D_{i}$ and the restriction of $D_{i}=\prod_{k \in \mathfrak{H}_{i}} Q_{k}$ to $\mathbb{R}^{n}$ is strictly positive on $\mathbb{R}^{n}$. Thus, $\left.B_{i}\right|_{\mathbb{R}^{n}}$ is $\left.P_{i}\right|_{\mathbb{R}^{n}}$ times a positive unit of $\mathcal{O}\left(\mathbb{R}^{n}\right)$.

STEP 2: ConstruCtion OF THE INFINITE SUM OF SQUARES $h$. In this step we construct the sum of squares of analytic functions $h$ announced in the reduction above. Fix an exhaustion of $\Omega$ by compact sets $\left\{K_{i}\right\}_{i \geq 1}$, that is, $K_{1} \neq \emptyset, K_{i} \subset$ $\operatorname{Int}_{\mathbb{C}^{n}}\left(K_{i+1}\right)$ and $\bigcup_{i \geq 1} K_{i}=\Omega$. For each $i \geq 1$, we set

$$
\mu_{i}=\sup _{K_{i}}\left|\frac{G_{0}^{2} \widehat{F}}{P_{i}}\right|^{2} \sum_{\ell \geq 1} \sup _{K_{i}}\left|B_{i \ell}{ }^{2}\right| \quad \text { and } \quad \gamma_{i}=\frac{1}{\sqrt{2^{i} \mu_{i}}} .
$$

For each $i \geq 1$, we have the following inequalities

$$
\sum_{\ell \geq 1} \sup _{K_{i}}\left|\gamma_{i} \frac{G_{0}^{2} \widehat{F}}{P_{i}} B_{i \ell}\right|^{2} \leq \gamma_{i}^{2} \sup _{K_{i}}\left|\frac{G_{0}^{2} \widehat{F}}{P_{i}}\right|^{2} \sum_{\ell \geq 1} \sup _{K_{i}}\left|B_{i \ell^{2}}\right| \leq \frac{1}{2^{i}} .
$$

Now, let $K$ be a compact subset of $\Omega$. As $\Omega \subset \bigcup_{i \geq 1} \operatorname{Int}_{\mathbb{C}^{n}}\left(K_{i}\right), K$ is contained in some $K_{i_{0}}$, hence in all $K_{i}$ for $i \geq i_{0}$, and so:

$$
\begin{aligned}
\sum_{i, \ell \geq 1} \sup _{K}\left|\gamma_{i} \frac{G_{0}^{2} \widehat{F}}{P_{i}} B_{i \ell}\right|^{2}= & \sum_{i=1}^{i_{0}-1} \sum_{\ell \geq 1} \sup _{K}\left|\gamma_{i} \frac{G_{0}^{2} \widehat{F}}{P_{i}} B_{i \ell}\right|^{2}+\sum_{i \geq i_{0}} \sum_{\ell \geq 1} \sup _{K}\left|\gamma_{i} \frac{G_{0}^{2} \widehat{F}}{P_{i}} B_{i \ell}\right|^{2} \\
\leq & \sum_{i=1}^{i_{0}-1} \sup _{K}\left|\gamma_{i} \frac{G_{0}^{2} \widehat{F}}{P_{i}}\right|^{2} \sum_{\ell \geq 1} \sup _{K}\left|B_{i \ell}{ }^{2}\right| \\
& +\sum_{i \geq i_{0}} \sum_{\ell \geq 1} \sup _{K_{i}}\left|\gamma_{i} \frac{G_{0}^{2} \widehat{F}}{P_{i}} B_{i \ell}\right|^{2} \\
& \leq \sum_{i=1}^{i_{0}-1} \sup _{K}\left|\gamma_{i} \frac{G_{0}^{2} \widehat{F}}{P_{i}}\right|^{2} \sum_{\ell \geq 1} \sup _{K}\left|B_{i \ell} \ell^{2}\right|+\sum_{i \geq i_{0}} \frac{1}{2^{i}}<+\infty .
\end{aligned}
$$

Consequently, we define the $\sigma$-invariant holomorphic function we were looking for as

$$
H=G_{0}^{4} \widehat{F}^{2}+\sum_{i, \ell \geq 1}\left(\gamma_{i} \frac{G_{0}^{2} \widehat{F}}{P_{i}} B_{i \ell}\right)^{2}
$$


hence, $h=\left.H\right|_{\mathbb{R}^{n}}$ is an infinite sum of squares of analytic functions on $\mathbb{R}^{n}$ and

$$
\{h=0\} \subset\left\{g_{0}=0\right\} \cup\{\widehat{f}=0\} \subset X .
$$

For our later purposes it is more convenient to have a different representation of $H$. Namely, let us check that

$$
H=G_{0}^{4} \widehat{F}^{2}+\sum_{i \geq 1}\left(\gamma_{i} \frac{G_{0}^{2} \widehat{F}}{P_{i}}\right)^{2} B_{i} .
$$

Indeed, since $B_{i}=\sum_{\ell \geq 1} B_{i \ell}^{2}$ for each $i \geq 1$, it is enough to check that the series $\sum_{i \geq 1}\left(\gamma_{i} \frac{G_{0}^{2} \widehat{F}}{P_{i}}\right)^{2} B_{i}$ converges in the sense of Definition 2.10(ii). Indeed, observe that for each $i, j \geq 1$, we have $\sup _{K_{j}}\left|B_{i}\right| \leq \sum_{\ell \geq 1} \sup _{K_{j}}\left|B_{i \ell}\right|^{2}$ and so, using ( ), we deduce

$$
\begin{aligned}
\sup _{K_{j}}\left|\gamma_{i} \frac{G_{0}^{2} \widehat{F}}{P_{i}}\right|^{2}\left|B_{i}\right| & =\sup _{K_{j}}\left|\gamma_{i} \frac{G_{0}^{2} \widehat{F}}{P_{i}}\right|^{2} \sup _{K_{j}}\left|B_{i}\right| \\
& \leq \gamma_{i}^{2} \sup _{K_{j}}\left|\frac{G_{0}^{2} \widehat{F}}{P_{i}}\right|^{2} \sum_{\ell \geq 1} \sup _{K_{j}}\left|B_{i \ell}\right| \leq \frac{1}{2^{i}} .
\end{aligned}
$$

Let $K$ be a compact subset of the open set $\Omega$ and let $i_{0} \geq 1$ be such that $K \subset K_{i}$ for $i \geq i_{0}$. We have

$$
\begin{aligned}
\sum_{i \geq 1} \sup _{K}\left|\gamma_{i} \frac{G_{0}^{2} \widehat{F}}{P_{i}}\right|^{2}\left|B_{i}\right| & =\sum_{i=1}^{i_{0}-1} \sup _{K}\left|\gamma_{i} \frac{G_{0}^{2} \widehat{F}}{P_{i}}\right|^{2}\left|B_{i}\right|+\sum_{i \geq i_{0}} \sup _{K}\left|\gamma_{i} \frac{G_{0}^{2} \widehat{F}}{P_{i}}\right|^{2}\left|B_{i}\right| \\
& \leq \sum_{i=1}^{i_{0}-1} \sup _{K}\left|\gamma_{i} \frac{G_{0}^{2} \widehat{F}}{P_{i}}\right|^{2} \sup _{K}\left|B_{i}\right|+\sum_{i \geq i_{0}} \frac{1}{2^{i}}<+\infty .
\end{aligned}
$$

STEP 3: CONSTRUCTION OF THE POSITIVE SEMIDEFINITE FUNCTION $q$ AND THE "DENOMINATOR" $g$. In this step we construct the functions $g, q$ announced in the reduction above and we show that everything holds.

Let us check first that $G_{0}^{2} \widehat{F}$ divides $H$ in $\mathcal{O}(\Omega)$. By $[8,4.4]$, it is enough to check that $G_{0}^{2} \widehat{F}$ divides each summand of $H$ on a neighborhood of $Z=\left\{G_{0}^{2} \widehat{F}=\right.$ $0\}=\bigcup_{k \geq 1} Z_{k}$ where $Z_{k}=\left\{G_{k}^{2} \widehat{F}_{k}=0\right\}$. Fix $i \geq 1$ and observe that since $B_{i}=P_{i} D_{i}$, we have

$$
\left(\gamma_{i} \frac{G_{0}^{2} \widehat{F}}{P_{i}}\right)^{2} B_{i}=\gamma_{i}^{2} \frac{G_{0}^{2} \widehat{F}}{P_{i}} \frac{G_{0}^{2} \widehat{F}}{P_{i}} B_{i}=G_{0}^{2} \widehat{F}\left(\gamma_{i}^{2} D_{i} \frac{G_{0}^{2} \widehat{F}}{P_{i}}\right) .
$$

Thus, there exists a holomorphic function $Q: \Omega \rightarrow \mathbb{C}$ such that $Q G_{0}^{2} \widehat{F}=H$. Of course, since $\Omega$ and $G_{0}, \widehat{F}, H$ are $\sigma$ invariant, so is $Q$; and, since $g_{0}^{2}=\left.G_{0}^{2}\right|_{\mathbb{R}^{n}}, \widehat{f}=$ 
$\left.\widehat{F}\right|_{\mathbb{R}^{n}}$ and $h=\left.H\right|_{\mathbb{R}^{n}}$ are positive semidefinite analytic functions, so is $q=\left.Q\right|_{\mathbb{R}^{n}}$. In fact, since $q g_{0}^{2} \widehat{f}=h$, we have $\{q=0\} \subset\{h=0\} \subset X$. Denote $g=q g_{0} \sqrt{u}$, where $u$ is the positive analytic unit satisfying $\widehat{f}=u f$ already constructed in STEP $\mathrm{I}$, and observe that

$$
g^{2} f=q^{2} g_{0}^{2} u f=q^{2} g_{0}^{2} \widehat{f}=q h \quad \text { and } \quad\{g=0\}=\left\{g_{0}=0\right\} \cup\{q=0\} \subset X
$$

Thus, we have got the required representation $g^{2} f=h q$ once we prove that

$$
\{q=0\} \subset \bigcup_{j, \ell \in I, j \neq \ell}\left(Y_{j} \cap Y_{\ell}\right) .
$$

Indeed, fix $i \geq 1$ and recall that $B_{i}=P_{i} D_{i}$, where $D_{i} \in \mathcal{O}(\Omega)$ is a holomorphic function on $\Omega$ whose restriction to $\mathbb{R}^{n}$ is a positive analytic unit. Moreover,

$$
H_{i}=\left(Q-\gamma_{i}^{2} D_{i} \frac{G_{0}^{2} \widehat{F}}{P_{i}}\right) G_{0}^{2} \widehat{F}=H-\left(\gamma_{i} \frac{G_{0}^{2} \widehat{F}}{P_{i}}\right)^{2} B_{i}=G_{0}^{4} \widehat{F}^{2}+\sum_{j \geq 1, j \neq i}\left(\gamma_{j} \frac{G_{0}^{2} \widehat{F}}{P_{j}}\right)^{2} B_{j} .
$$

is a $\sigma$-invariant holomorphic function on $\Omega$ whose restriction to $\mathbb{R}^{n}$ is an infinite sum of squares of analytic functions on $\mathbb{R}^{n}$. Write $Q_{i}^{\prime}=Q-\gamma_{i}^{2} D_{i} \frac{G_{0}^{2} \widehat{F}}{P_{i}}$ which is a $\sigma$-invariant holomorphic function on $\Omega$ such that

$$
H_{i}=Q_{i}^{\prime} G_{0}^{2} \widehat{F} \quad \text { and } \quad Q=Q_{i}^{\prime}+\gamma_{i}^{2} D_{i} \frac{G_{0}^{2} \widehat{F}}{P_{i}} .
$$

Since the restrictions to $\mathbb{R}^{n}$ of $H_{i}, G_{0}^{2}, \widehat{F}$ are positive semidefinite also the restriction of $Q_{i}^{\prime}$ to $\mathbb{R}^{n}$ is positive semidefinite and so

$$
\{q=0\} \subset\left\{\frac{G_{0}^{2} \widehat{F}}{P_{i}}\right\} \cap \mathbb{R}^{n}=\bigcup_{k \notin \mathfrak{H}_{i}} X_{k} .
$$

Now, if $i \notin I$, that is, if $\mathfrak{F}_{i}$ is a finite set, we have that $\mathfrak{H}_{i}=\mathfrak{F}_{i}$ and

$$
Y_{i} \cap\{q=0\} \subset Y_{i} \cap \bigcup_{k \notin \mathfrak{H}_{i}} X_{k}=\varnothing ;
$$

hence, $\{q=0\} \cap \bigcup_{j \notin I} Y_{j}=\varnothing$. Next, if $i \in I$, then $\mathfrak{H}_{i}=\mathfrak{G}_{i}=\left\{k \geq 1: Y_{i} \subset X_{k}\right\}$ and

$$
Y_{i} \cap\{q=0\} \subset Y_{i} \cap\left(\left(\bigcup_{k \notin \mathfrak{H}_{i}} X_{k}\right) \backslash \bigcup_{j \notin I} Y_{j}\right) \subset Y_{i} \cap \bigcup_{j \in I, j \neq i} Y_{j} .
$$

Putting all together, we deduce

$\{q=0\}=X \cap\{q=0\}=\bigcup_{i \geq 1} Y_{i} \cap\{q=0\}=\bigcup_{i \in I} Y_{i} \cap\{q=0\} \subset \bigcup_{i, j \in I, i \neq j} Y_{i} \cap Y_{j}$, and we are done. 


\section{Applications to Hilbert's 17th Problem}

We begin this section with the construction of the irreducible factors of analytic and holomorphic functions.

\subsection{Irreducible factors of a holomorphic function}

It has been well-known for long (see [22] and [9]) that every (complex) analytic subset $Z$ of an open set $\Omega \subset \mathbb{C}^{n}$ can be uniquely decomposed into a locally finite family of irreducible analytic sets, which are called the irreducible components of $Z$. Here an analytic subset $Y$ of $\Omega$ is irreducible if $Y$ is not the union of two analytic subsets $Y_{1}, Y_{2}$ of $\Omega$ both different from $Y$. Analogously, a holomorphic function $F \in \mathcal{H}(\Omega)$ is irreducible if it cannot be written as the product of two holomorphic functions on $\Omega$ with nonempty zeroset. Given holomorphic functions $G, F \in \mathcal{H}(\Omega)$, we say that $G$ divides $F$ with multiplicity $k \geq 0$ if $G^{k}$ divides $F$ but $G^{k+1}$ does not; of course, taking germs at any point of $\Omega$ at which both $F$ and $G$ vanish one can always compute this multiplicity. In case $H^{2}(\Omega, \mathbb{Z})=0$ (for instance, when it retracts to $\mathbb{R}^{n}$ ) the decomposition into irreducible components of (complex) analytic subsets of $\Omega$ provides a procedure to decompose each holomorphic function $F \in \mathcal{H}(\Omega)$ as the sheaf-product of its irreducible factors. Indeed, let $Z=\{F=0\}$ and let $\left\{Z_{k}\right\}_{k \geq 1}$ be the irreducible components of $Z$. Now, since $\operatorname{dim} Z_{k}=n-1$, for each $z \in Z_{k}$ there exists a holomorphic function germ $\xi_{k, z} \in \mathcal{O}_{\Omega, z}$ that generates the ideal of the analytic germ $Z_{k, z}$ in $\Omega_{z}$. Consider the locally principal coherent analytic sheaf $\mathcal{J}_{k}$ of ideals

$$
\mathcal{J}_{k, z}= \begin{cases}\xi_{k, z} \mathcal{O}_{\Omega, z} & \text { if } z \in Z_{k}, \\ \mathcal{O}_{\Omega, z} & \text { if } z \in \Omega \backslash Z_{k}\end{cases}
$$

which is therefore globally principal and is generated by a function $H_{k} \in \mathcal{H}(\Omega)$. For each $k \geq 1$ let $m_{k}$ denote the multiplicity of division of $F$ by $H_{k}$. A straighforward computation shows that $F=\pi H_{k}^{m_{k}}$. We call $\left\{H_{k}\right\}_{k \geq 1}$ the collection of the irreducible factors of $F$.

The factorization of a real analytic functions is similar but quite more involving.

\subsection{Irreducible factors of an analytic function}

We say that an analytic function $f \in \mathcal{O}(W)$ on an open set $W \subset \mathbb{R}^{n}$ is irreducible if it cannot be written as the product of two analytic functions with nonempty zeroset. Similarly, given analytic functions $g, f \in \mathcal{O}\left(\mathbb{R}^{n}\right)$, we say that $g$ divides $f$ with multiplicity $k \geq 0$ if $g^{k}$ divides $f$ but $g^{k+1}$ does not; again such a $k$ exists and it is finite.

Given a closed set $Z \subset \mathbb{C}^{n}$, germs (of sets or of holomorphic functions) at $Z$ are defined exactly as germs at a point, through neighborhoods of $Z$ in $\mathbb{C}^{n}$; we denote by $F_{Z}$ the germ at $Z$ of a holomorphic function $F$ defined in some neighborhood of $Z$. If $F \in \mathcal{H}(\Omega)$ is a $\sigma$-invariant, $\mathbb{R}^{n} \subset \Omega$ and $Z=\mathbb{R}^{n}$, then the germ $F_{\mathbb{R}^{n}}$ 
can be identified with the function $f:=\left.F\right|_{\mathbb{R}^{n}}$, because each of them determines uniquely the other one.

We define the irreducible factors of $f \in \mathcal{O}\left(\mathbb{R}^{n}\right)$ as follows. Let $F: \Omega \rightarrow \mathbb{C}$ be a holomorphic extension of $f$ to an open neighborhood $\Omega$ of $\mathbb{R}^{n}$ in $\mathbb{C}^{n}$. By $[22,6$. Prop.9], there exists a unique locally finite family of irreducible germs $\left\{Y_{k}\right\}_{k \geq 1}$ at $\mathbb{R}^{n}$ such that $Y_{j} \not \subset Y_{k}$ if $j \neq k$ and $\{F=0\}_{\mathbb{R}^{n}}=\bigcup_{k \geq 1} Y_{k}$. By [22, 6 . Prop.8, Cor.2], for each $k \geq 1$ there exists a $\sigma$-invariant contractible open Stein neighborhood $\Omega_{k}$ of $\mathbb{R}^{n}$ in $\bar{C}^{n}$ and an irreducible analytic set $Z_{k}$ in $\Omega_{k}$ such that $Z_{k, \mathbb{R}^{n}}=Y_{k}$. Once more, since $\operatorname{dim} Z_{k}=n-1$, there exists $H_{k} \in \mathcal{H}\left(\Omega_{k}\right)$ which generates the ideal $\mathcal{J}_{\Omega_{k}}\left(Z_{k}\right)$ of holomorphic functions on $\Omega_{k}$ vanishing identically on $Z_{k}$. Notice that if $Z_{k}$ is $\sigma$-invariant, we may further assume, by Lemma 2.2, that $H_{k}$ is also $\sigma$-invariant. On the other hand, if $Z_{k}$ is not $\sigma$-invariant, then $\overline{H_{k} \circ \sigma}$ divides $F$ in $\Omega_{k}$ with the same multiplicity as $H_{k}$. For each $k \geq 1$, we define

$$
F_{k}=\left\{\begin{array}{cl}
H_{k} & \text { if } Z_{k} \text { is } \sigma \text {-invariant } \\
H_{k} \overline{H_{k} \circ \sigma} & \text { if } Z_{k} \text { is not } \sigma \text {-invariant }
\end{array}\right.
$$

Observe that $f_{k}=\left.F_{k}\right|_{\mathbb{R}^{n}} \in \mathcal{O}\left(\mathbb{R}^{n}\right)$ is irreducible and divides $f$ for all $k \geq 1$. We eliminate the repetitions and denote again by $\left\{f_{k}\right\}_{k \geq 1}$ the collection of the previous irreducible factors. Denote by $m_{k}$ the multiplicity of division of $f$ by $f_{k}$. A straighforward computation shows that $f=\pi f_{k}^{m_{k}}$. We call $\left\{f_{k}\right\}_{k \geq 1}$ the collection of the real irreducible factors of $f$.

Definition 6.1. We focus a special attention in a particular type of irreducible factor. Following [8], we say that $f_{k}$ is a special factor of $f$ if:

(i) The germ $Z_{k, \mathbb{R}^{n}}$ is $\sigma$-invariant,

(ii) $f_{k}$ divides $f$ with odd multiplicity, and

(iii) The dimension $d$ of $\left\{f_{k}=0\right\}$ satisfies $1 \leq d \leq n-2$.

Of course, we may always assume that the special factors $f_{k}$ of $f$ are positive semidefinite analytic functions. The reason for asking that the dimension of the zeroset of a special factor is strictly positive is the following: as we know, an irreducible factor $f_{k}$ of $f$ satisfying conditions (i) and (ii) above and whose zero set has dimension zero is a finite sum of squares (use once more [5, Theorem 1]); hence, it produces no obstruction to represent $f$ as an either finite or infinite sum of squares.

Contrary to what happens in the complex case the zeroset of a special factor can be reducible as a real global analytic set; see $[8,2.7]$ or the example below.

Example 6.2. For each $k \geq 0$ consider the polynomial functions on $\mathbb{R}^{2}$ and $\mathbb{R}^{3}$ respectively given by the formulae $f_{k}(x, y)=(x-k)^{2}+y^{2}-1$ and $g_{k}(x, y, z)=$ $z+f_{k}$. Let $f: \mathbb{R}^{2} \rightarrow \mathbb{R}$ and $g: \mathbb{R}^{3} \rightarrow \mathbb{R}$ be the sheaf-products of the $f_{k}$ 's and the $g_{k}^{\prime} s$ respectively. For each $k, \ell \geq 0$ with $\ell \neq k$, we have $\left\{f_{k}=0\right\} \cap\left\{f_{\ell}=0\right\} \neq \varnothing$ if and only if $|\ell-k| \leq 1$; and, if such is the case, the two intersection points of $\left\{f_{k}=0\right\}$ and $\left\{f_{\ell}=0\right\}$ are

$$
p_{\ell, k, \pm}=\left(\frac{\ell+k}{2}, \pm \frac{\sqrt{3-(k-\ell)^{2}}}{2}\right) .
$$


For each real number $M>0$, consider the analytic function

$$
h_{M}: \mathbb{R}^{3} \rightarrow \mathbb{R},(x, y, z) \mapsto g^{2}+z^{4}+M^{2} f^{2}(z) .
$$

Observe that $\left\{h_{M}=0\right\}=\bigcup_{k \geq 0}\left\{(x, y, 0):(x-k)^{2}+y^{2}-1\right\}$, which is a countable locally finite union of circunferences, which are compact analytic subsets of $\mathbb{R}^{3}$. Observe that for each $k, \ell \geq 0$ with $k \neq \ell$ and $|\ell-k| \leq 1$, the initial form of the germ of $h_{M}\left((x, y, z)+q_{\ell, k, \pm}\right)$ at $q_{\ell, k, \pm}=\left(p_{\ell, k, \pm}, 0\right)$ is a homogeneous polynomial of the type

$$
P_{\ell, k, \pm}=\alpha^{2}(z+u)^{2}(z+v)^{2}+z^{4}+M \beta^{2} u^{2} v^{2}
$$

where $u=(\ell-k) x \pm \sqrt{3-(k-\ell)^{2}} y$ and $v=-(\ell-k) x \pm \sqrt{3-(k-\ell)^{2}} y$ and $\alpha, \beta$ are nonzero real numbers depending of $q_{\ell, k, \pm}$. By $[8,2.6]$, we may choose $M>0$ in such a way that all the homogeneous polynomials $P_{\ell, k, \pm}$ are irreducible. For this choice of $M$ one can prove, following the same ideas developed in $[8,2.7]$, that $h_{M}$ is a special factor.

\subsection{Applications}

Now, we apply all we have learnt concerning factorization of analytic functions to localize the obstruction to represent a positive semidefinite analytic function as an either finite or infinite sum of squares.

Lemma 6.3. Let $f: \mathbb{R}^{n} \rightarrow \mathbb{R}$ be a positive semidefinite analytic function. Then, $f=g h$ where $g$ is a finite sum of squares and $h$ is a square free positive semidefinite analytic function whose irreducible factors are the special factors of $f$. In fact, $g$ is a sum of $2^{n+1}$ squares.

Proof. First, let $\left\{h_{k}\right\}_{k \geq 1}$ be the special factors of $f$ and let $\left\{g_{j}\right\}_{j \geq 1}$ be all the others irreducible factors. Denote $2 n_{k}+1$ the multiplicity of division of $f$ by $h_{k}$ and $m_{j}$ be the multiplicity of division of $f$ by $g_{j}$. Notice that if $m_{j}$ is odd and $\left\{g_{j}=0\right\}$ is not a singleton, then there exists a $\sigma$-invariant open neighborhood $\Omega_{j}$ of $\mathbb{R}^{n}$ in $\mathbb{C}^{n}$, a holomorphic extension $G_{j}$ of $g_{j}$ to $\Omega_{j}$ and an (irreducible) holomorphic function $H_{j} \in \mathcal{H}\left(\Omega_{j}\right)$ such that $G_{j}=H_{j} \overline{H_{j} \circ \sigma}$. Thus, in particular $g_{j}$ and so $g_{j}^{m_{j}}$ are sums of two squares in $\mathcal{O}\left(\mathbb{R}^{n}\right)$. On the other hand, if $\left\{g_{j}=0\right\}$ has dimension zero (and so it is a point) we claim that $g_{j}$ and so $g_{j}^{m_{j}}$ are sums of $2^{n+1}$ squares (with controlled denominators). Indeed, if $\left\{g_{j}=0\right\}=\{a\}$, the germ $g_{j, a}$ is a sum of $p=2^{n}+n$ squares (because a suitable modification of the germ is algebraic, see [5, 418-420]). In fact, by means of $[16,7.4]$ such representation may be achieved with controlled denominators. Now, the claim follows from Theorem 2.12.

Next, consider the sheaf-product $g=\left(\pi h_{k}^{n_{k}}\right)^{2}\left(\pi g_{j}^{m_{j}}\right)$ and observe that, by Theorem 3.1, $g$ is a sum of $2^{n+1}$ squares. Next, let $h=\pi h_{k}$ and observe that we may assume that $f=g h$. Clearly, the irreducible factors of $h$ are the special factors of $f$, and we are done. 
Remark 6.4. If $n=2$ it is clear that the zero sets of the irreducible factors have either dimension 0 or 1 . Of course, if $f$ is positive semidefinite, the zero sets of the irreducible factors $g_{j}$ which divide $f$ with odd multiplicity have dimension 0 , and so they are singletons $\left\{a_{j}\right\}$. In fact, in [6, III.Lemme 7(a)] it is proved that the germ $g_{j, a_{j}}$ is a sum of two squares. This implies that there exists a $\sigma$-invariant open neighborhood $\Omega_{j}$ of $\mathbb{R}^{2}$ in $\mathbb{C}^{2}$, a holomorphic extension $G_{j}$ of $g_{j}$ to $\Omega$ and an (irreducible) holomorphic function $H_{j} \in \mathcal{H}\left(\Omega_{j}\right)$ such that $G_{j}=H_{j} \overline{H_{j} \circ \sigma}$. Thus, in particular $g_{j}$ is a sum of two squares in $\mathcal{O}\left(\mathbb{R}^{n}\right)$, and so, by Theorem 3.1 and Remark 3.4(ii), one deduces that $f$ is a sum of two squares.

Moreover, the previous discussion proves that the special factors only appear for $n \geq 3$. Hence, obstructions for a positive answer to $\mathrm{H} 17$ and $\mathrm{H} 17_{\infty}$ can only arise for $n \geq 3$ and, of course, they are focused on the special factors.

Proof of Theorem 1.4. First, by Lemma 6.3, we write $f=g h$ where $g$ is a finite sum of squares and $h$ is a square free positive semidefinite analytic function whose irreducible factors are the special factors of $f$. Since the zero set of $f$ is the union of a discrete set and a compact set, it follows that the zero set of $h$ is compact; hence, $h$ is, by $[14,20]$, a finite sum of squares and so also $f$ is a finite sum of squares, as wanted.

In this framework, Theorems 1.1, 1.2 and 1.3 together with Lemma 6.3 provide the following results.

Corollary 6.5. Let $f: \mathbb{R}^{n} \rightarrow \mathbb{R}$ be a positive semidefinite analytic function and let $\left\{h_{k}\right\}_{k \geq 1}$ be its special factors. We have:

(i) If each $h_{k}$ is a sum of $2^{r}$ squares at $\left\{h_{k}=0\right\}$, then $f$ is a sum of $2^{n+r}$ squares (with controlled denominators).

(ii) If each $h_{k}$ is a finite sum of squares at $\left\{h_{k}=0\right\}$, then $f$ is an infinite sum of squares.

(iii) If each $h_{k}$ is an infinite sum of squares at $\left\{h_{k}=0\right\}$, there is a positive semidefinite $q \in \mathcal{O}\left(\mathbb{R}^{n}\right)$ such that $\{q=0\} \subset\{f=0\}, \operatorname{dim}\{q=0\}<\operatorname{dim}\{f=0\}$ and $q f$ is an infinite sum of squares.

Corollary 6.6. Let $f: \mathbb{R}^{n} \rightarrow \mathbb{R}$ be a positive semidefinite analytic function and let $\left\{h_{k}\right\}_{k \geq 1}$ be its special factors. We have:

(i) If each $h_{k}$ is of Nash type, then $f$ is a sum of $2^{n+1}$ squares (with controlled denominators).

(ii) If each $\left\{h_{k}=0\right\}$ is either compact or of Nash type, then $f$ is an infinite sum of squares.

\subsection{Pfister's formulae for arbitrary affine analytic manifolds}

Let us show how to extend Pfister's formulae (see Theorem 1.1) to an affine analytic manifold $M$ of dimension $d$. First, we embed $M$ as a closed set in $\mathbb{R}^{n}$ for $n=2 d+1$ 
(see [17, 2.15.12]); hence, there is an analytic function $h: \mathbb{R}^{n} \rightarrow \mathbb{R}$ with zero set $\{h=0\}=M$. Consider an open tubular neighborhood $U$ of $M$ in $\mathbb{R}^{n}$, endowed with the corresponding analytic retraction $\pi: U \rightarrow M$. By composition with $\pi$, all functions extend from $M$ to $U$. Moreover, if $f \in \mathcal{O}(M)$ is positive semidefinite, then so is $f^{\prime}=(f \circ \pi)+\left.h\right|_{U} ^{2}$ and its zero set $\left\{f^{\prime}=0\right\}=\{f=0\}$ is a closed subset of $M$. In addition, if $f \in \mathcal{O}(M)$ is a sum of $p$ squares at $\{f=0\}$, then $f^{\prime}=(f \circ \pi)+\left.h\right|_{U} ^{2}$ is a sum of $p+1$ squares at $\left\{f^{\prime}=0\right\}=\{f=0\}$. Hence, we can generalize the statements already proposed in Theorems 1.1,1.2 and 1.3 for an arbitrary affine analytic manifold $M$ of dimension $d$, just changing $n$ by $2 d+1$. If in addition $H^{1}\left(M, \mathbb{Z}_{2}\right)=H^{2}(M, \mathbb{Z})=0$, analogous factorization results to the ones for $\mathbb{R}^{n}$ hold for $M$, and so we can generalize Corollaries 6.5 and 6.6 for $M$ changing again $n$ by $2 d+1$.

\section{References}

[1] F. Acquistapace, F. Broglia and J. F. Fernando, On real Nullstellensatz and Lojasiewicz's radical ideal in the global analytic setting, preprint RAAG (2014), arXiv: 1207.0391.

[2] F. Acquistapace, F. Broglia, J. F. Fernando and J. M. Ruiz, On the finiteness of Pythagoras numbers of real meromorphic functions, Bull. Soc. Math. France 138 (2010), 291-307.

[3] E. ARTIN, Über die Zerlegung definiter Funktionen in Quadrate, Hamb. Abh. 5 (1927), 100-115, In: "The collected papers of Emil Artin", Addison-Wesley, Reading 1965, $273-$ 288.

[4] J. Bochnak, M. Coste and M. F. RoY, "Real Algebraic Geometry", translated from the 1987 French original, revised by the authors, Ergebn. der Math. und ihrer Grenzgebiete (3), Vol. 36, Springer-Verlag, Berlin, 1998.

[5] J. Bochnak, W. KUCHARZ and M. Shiota, On equivalence of ideals of real global analytic functions and the 17th Hilbert problem, Invent. Math. 63 (1981), 403-421.

[6] J. BOCHNAK and J. J. RISLER, Le théorème des zéros pour les variétés analytiques réelles de dimension 2, Ann. Sci. Éc. Norm. Supér. (4) 8 (1975), 353-363.

[7] H. CARTAN, Variétés analytiques réelles et variétés analytiques complexes, Bull. Soc. Math. France 85 (1957), 77-99.

[8] J. F. FERNANDO, On Hilbert's 17th problem for global analytic functions in dimension 3, Comment. Math. Helv. 83 (2008), 67-100.

[9] O. Forster, Primärzerlung in Steinschen Algebren, Math. Ann. 154 (1964), 307-329.

[10] H. GRAUERT, Analytische Faserungen über holomorph-vollständigen Räumen, Math. Ann. 135 (1958), 263-273.

[11] R. Gunning and H. Rossi, "Analytic Functions of Several Complex Variables", Prentice Hall, Englewood Cliff, 1965.

[12] E. Hille and R. S. Phillips, "Functional Analysis and Semigroups", Amer. Math. Soc. Colloq. Publ., Vol. 31 (rev. ed.), Amer. Math. Soc., Providence, RI, 1957.

[13] P. JaWORSKi, Positive definite analytic functions and vector bundles, Bull. Ad. Pol. Sci., Sér. Sci. Math. 30 (1982), 501-506.

[14] P. JAWORSKI, Extensions of orderings on fields of quotients of rings of real analytic functions, Math. Nachr. 125 (1986), 329-339.

[15] P. JAWORSKI, The 17th Hilbert problem for noncompact real analytic manifolds, In: "Real Algebraic Geometry" (Rennes, 1991), Lecture Notes in Math., Vol. 1524, Springer, Berlin, 1992, 289-295. 
[16] L. MAHÉ, Level and Pythagoras number of some geometric rings, Math. Z. 204 (1990), 615-629.

[17] R. NARASimhan, "Analysis on Real and Complex Manifolds" (2 $2^{\text {nd }}$ ed.), Advanced Studies in Pure Mathematics, Vol. 1, Masson \& Cie, Éditeur, Paris; North-Holland Publishing Co., Amsterdam-London; American Elsevier Publishing Co., Inc., New York, 1973.

[18] A. PFISTER, Zur Darstellung von -1 als Summe von Quadraten in einem Körper, J. London Math. Soc. 40 (1965), 159-165.

[19] W. Rudin, "Real and Complex Analysis", McGraw-Hill Book Co., New York, 1987.

[20] J. M. RUIZ, On Hilbert's 17th problem and real Nullstellensatz for global analytic functions, Math. Z. 190 (1985), 447-454.

[21] M. SHIOTA, Sur la factorialité de l'anneau des fonctions analytiques, C. R. Acad. Sci. Paris, Sér. A 285 (1977), 253- 255.

[22] H. WhitNEY and F. BRUHAT, Quelques propriétés fondamentales des ensembles analytiques réels, Comment. Math. Helv. 33 (1959), 132-160.

[23] M. G. ZaIdenberg, S. G. Kre`n, P. A. Kučment and A. A. Pankov, Banach bundles and linear operators (Russian), Uspehi Mat. Nauk 30 (1975), (185), 101-157. English translation: Russian Math. Surveys 30 (1975), 115-175.

Dipartimento di Matematica Università degli Studi di Pisa Largo Bruno Pontecorvo, 5 56127 Pisa, Italia acquistf@dm.unipi.it broglia@dm.unipi.it

Departamento de Álgebra Facultad de Ciencias Matemáticas Universidad Complutense de Madrid 28040 Madrid, Spain josefer@mat.ucm.es 